TRANSACTIONS OF THE

AMERICAN MATHEMATICAL SOCIETY

Volume 365, Number 3, March 2013, Pages 1219-1249

S 0002-9947(2012)05573-5

Article electronically published on October 31,2012

\title{
RESIDUATED FRAMES WITH APPLICATIONS TO DECIDABILITY
}

\author{
NIKOLAOS GALATOS AND PETER JIPSEN
}

\begin{abstract}
Residuated frames provide relational semantics for substructural logics and are a natural generalization of Kripke frames in intuitionistic and modal logic, and of phase spaces in linear logic. We explore the connection between Gentzen systems and residuated frames and illustrate how frames provide a uniform treatment for semantic proofs of cut-elimination, the finite model property and the finite embeddability property, which imply the decidability of the equational/universal theories of the associated residuated lattice-ordered groupoids. In particular these techniques allow us to prove that the variety of involutive FL-algebras and several related varieties have the finite model property.
\end{abstract}

Substructural logics and their algebraic formulation as varieties of residuated lattices and FL-algebras provide a general framework for a wide range of logical and algebraic systems, such as

Classical propositional logic

Intuitionistic logic

$\leftrightarrow \quad$ Boolean algebras

Lukasiewicz logic

$\leftrightarrow \quad$ Heyting algebras

Abelian logic

$\leftrightarrow$ MV-algebras

Basic fuzzy logic

$\leftrightarrow$ abelian lattice-ordered groups

Monoidal $t$-norm logic

$\leftrightarrow$ BL-algebras

$\leftrightarrow \quad$ MTL-algebras

Noncommutative mult. add. linear logic $\leftrightarrow$ InFL-algebras

Full Lambek calculus

$\leftrightarrow \quad$ FL-algebras

and lattice-ordered groups, symmetric relation algebras and many other systems.

In this paper we introduce residuated frames and show that they provide relational semantics for substructural logics and representations for residuated structures. Our approach is driven by the applications of the theory. As is the case with Kripke frames for modal logics, residuated frames provide a valuable tool for solving both algebraic and logical problems. Moreover we show that there is a direct link between Gentzen-style sequent calculi and our residuated frames, which gives insight into the connection between a cut-free proof system and the finite embeddability property for the corresponding variety of algebras.

After an overview of residuated structures and certain types of closure operators called nuclei, we define residuated frames and Gentzen frames (Section 2), and provide several examples. We then prove a general homomorphism theorem in the

Received by the editors August 29, 2008 and, in revised form, February 22, 2011.

2010 Mathematics Subject Classification. Primary 06F05; Secondary 08B15, 03B47, 03G10, $03 \mathrm{~F} 05$.

Key words and phrases. Substructural logic, Gentzen system, residuated lattice, residuated frame, cut elimination, decidability, finite model property, finite embeddability property.

(C)2012 American Mathematical Society

Reverts to public domain 28 years from publication 
setting of Gentzen frames (Theorem 2.6) and apply it to the particular examples. Results in Section 3 include the cut-elimination property for several logical systems, the decidability of logics and of varieties of residuated structures, the finite model property, and the finite embeddability property. The homomorphism theorem generalizes and simplifies ideas found in several papers $2,4,19,20,24,26,27$. that address the above types of problems in otherwise seemingly unrelated ways. Thus the notion of a residuated frame provides a unifying framework for the analysis of various logical and algebraic properties and for their proof in a general setting.

Ono and Komori 20], and later Okada and Terui [19], prove cut-elimination and decidability for the full Lambek calculus (and other systems) using monoid semantics and phase spaces. Blok and van Alten [3, 4] prove the finite embeddability property for several classes of residuated structures using a combinatorial argument, and Belardinelli, Jipsen, Ono 2] and Wille 26] give algebraic proofs of cut elimination and decidability for FL-algebras and involutive residuated lattices.

We present a common generalization of these results, and use it to prove several new results. In particular, we consider all subvarieties of residuated latticeordered unital groupoids ( $r \ell u$-groupoids) defined by an equation using the symbols $\{\vee, \cdot 1\}$ and prove cut elimination for sequent calculi associated with these equational classes (Corollary 3.13). We apply this result to obtain the finite model property for many of these classes (essentially those where the defining equation corresponds to a sequent rule whose premises are no more complex than its conclusion; see Theorem [3.15). For integral $r$ lu-groupoids we are able to prove the stronger finite embeddability property for all subvarieties defined by a $\{\vee, \cdot, 1\}$ equation (Theorem 3.18), which implies that the universal theory of each of these classes is decidable. Note that Farulewski [1] and Buszkowski [7] prove the finite embeddability property for all residuated groupoids, all distributive lattice-ordered residuated groupoids and all lattice-ordered residuated groupoids with Boolean or intuitionistic negation.

In Sections 4 and 5 we adapt our techniques to involutive residuated structures, and prove new results about them, including the finite model property of involutive FL-algebras and its generalizations (Corollary [5.7, 5.9). This extends the cutelimination results of Abrusci [1, and the decidability of cyclic FL-algebras proved by Yetter [27] and Wille [26].

Similar generalized Kripke frames have been introduced and applied to residuated structures independently by Gehrke [17] and in algebraic form by Dunn, Gehrke and Palmigiano [10. The connections of this approach to ours are discussed in more detail after the definition of residuated frames in Section 2. Our somewhat more general perspective is required to establish the fundamental link between Gentzen sequent calculi and residuated frames.

\section{Residuated structures AND NUClei}

A residuated lattice, in the generality of [6], is of the form $\mathbf{A}=(A, \wedge, \vee, \cdot, \backslash, /, 1)$, where $(A, \wedge, \vee)$ is a lattice, $(A, \cdot, 1)$ is a monoid and residuation holds:

(res) $\quad$ for all $x, y, z \in A \quad x y \leq z \quad$ iff $\quad x \leq z / y \quad$ iff $\quad y \leq x \backslash z$.

Here $\leq$ denotes the lattice order and $x y$ stands for $x \cdot y$. The operations of $\mathbf{A}$ are called meet, join, multiplication, left and right division, and multiplicative unit, respectively. Residuation (res) can be reformulated in equational form [6], so the 
class $\mathrm{RL}$ of residuated lattices is a variety. We recall some basic results on residuated lattices; for more on residuated structures we refer the reader to [18] and [12].

Lemma 1.1. Multiplication preserves existing joins, and divisions preserve existing meets in the numerator and convert existing joins in the denominator to meets.

An FL-algebra is a residuated lattice expanded with an arbitrary constant 0 . We denote the variety of FL-algebras by FL. Of special importance are residuated lattices (and FL-algebras) that satisfy the equations $x y=y x, x \leq 1$ and $x \leq x^{2}$. They are called commutative, integral and contractive, respectively. Note that commutativity implies $x \backslash y=y / x$, and in this case $x \rightarrow y$ is used for the common value. The constant 0 allows for the definition of two negation operations $\sim x=x \backslash 0$ and $-x=0 / x$. An FL-algebra is called involutive (InFL-algebra) if it satisfies the equations $\sim-x=x=-\sim x$; InFL denotes the corresponding variety. A cyclic InFLalgebra satisfies $\sim x=-x$. Note that cyclicity is a consequence of commutativity.

Many subsequent results apply to more general residuated structures that are not assumed to be lattice-ordered, associative or have a unit element, which leads to the following definitions. A pogroupoid is a structure $\mathbf{G}=(G, \leq, \cdot)$, where $\leq$ is a partial order on $G$ and the binary operation - is order preserving. A residuated groupoid is a structure $\mathbf{G}=(G, \leq, \cdot, \backslash, /)$ where $\leq$ is a partial order on $G$ and the residuation property (res) holds. It follows that multiplication is order preserving. If $\leq$ is a lattice order, then $(G, \wedge, \vee, \cdot, \backslash, /)$ is said to be an $r \ell$-groupoid, and if this algebra is extended with a constant 1 that is a multiplicative unit, or with an arbitrary constant 0 , then it is said to be an rlu-groupoid or an $r \ell z$-groupoid, respectively. Note that a residuated lattice is an associative $r \ell u$-groupoid, and an FL-algebra is an associative $r \ell u z$-groupoid. Involutive rlu-groupoids are defined like InFLalgebras, but without assuming associativity. The varieties of $r \ell u(z)$-groupoids and involutive $r \ell u$-groupoids are denoted by $\mathrm{RLU}(Z) \mathrm{G}$ and $\mathrm{InGL}$, respectively.

Galois connections. For posets $\mathbf{P}$ and $\mathbf{Q}$, the maps ${ }^{\triangleright}: P \rightarrow Q$ and $\triangleleft: Q \rightarrow P$ forms a Galois connection if for all $p \in P$ and $q \in Q, q \leq p^{\triangleright}$ iff $p \leq q^{\triangleleft}$. A closure operator $\gamma$ on $\mathbf{P}$ is an increasing, monotone and idempotent map, i.e., $x \leq \gamma(x)$, $x \leq y$ implies $\gamma(x) \leq \gamma(y)$, and $\gamma(\gamma(x))=\gamma(x)$, for all $x, y \in P$. $\mathbf{P}_{\gamma}$ denotes the poset of $\gamma$-closed sets, with underlying set the image $P_{\gamma}=\gamma[P]=\{\gamma(p): p \in P\}$.

Given a relation $R \subseteq A \times B$ between sets $A, B$, for $X \subseteq A$ and $Y \subseteq B$ define

$$
X R Y \quad \text { iff } \quad x R y \text { for all } x \in X, y \in Y \text {. }
$$

We also abbreviate $\{x\} R Y$ and $X R\{y\}$ as $x R Y$ and $X R y$. Note that a pair of maps ${ }^{\triangleright}: \mathcal{P}(A) \rightarrow \mathcal{P}(B)$ and ${ }^{\triangleleft}: \mathcal{P}(B) \rightarrow \mathcal{P}(A)$ forms a Galois connection iff $X^{\triangleright}=\{y: X R y\}$ and $Y^{\triangleleft}=\{x: x R Y\}$, for some relation $R \subseteq A \times B$. In this case we have $x R y$ iff $x \in\{y\}^{\triangleleft}$ (iff $y \in\{x\}^{\triangleright}$ ) and $(\triangleright, \triangleleft)$ is called the Galois connection induced by $R$. The closure operator $\gamma_{R}: \mathcal{P}(A) \rightarrow \mathcal{P}(A)$ associated with $R$ is $\gamma_{R}(X)=X^{\triangleright \triangleleft}$. For a closure operator $\gamma$ on a complete lattice $\mathbf{P}, D \subseteq P$ is a basis for $\gamma$ if the elements in $\gamma[P]$ are exactly the meets of elements of $D$. Note that $D \subseteq \gamma[P]$. The interior operator in a topological space is a closure operator under the dual order, so this notion of a basis is equivalent to the usual one in topology.

Lemma 1.2. Let $A$ and $B$ be sets.

(i) If $R$ is a relation between $A$ and $B$, then $\gamma_{R}$ is a closure operator on $\mathcal{P}(A)$.

(ii) If $\gamma$ is a closure operator on $\mathcal{P}(A)$, then $\gamma=\gamma_{R}$ for some $R$ with domain A. 
(iii) If $\triangleright, \triangleleft$ is the Galois connection induced by $R \subseteq A \times B$, then $\{\{b\} \triangleleft: b \in B\}$ is a basis for $\gamma_{R}$.

(iv) For a closed set $X \subseteq A$ and $a \in A$ we have $a \in X$ if and only if for all $b \in B, X \subseteq\{b\}^{\triangleleft} \Rightarrow a \in\{b\}^{\triangleleft}$.

Nuclei. A nucleus on a pogroupoid G (originally defined in the context of Brouwerian algebras [22] and quantales 21]) is a closure operator $\gamma$ on (the poset reduct of) $\mathbf{G}$ such that $\gamma(x) \gamma(y) \leq \gamma(x y)$ [equivalently $\gamma(\gamma(x) \gamma(y))=\gamma(x y)$ ] for all $x, y \in G$.

Lemma $1.3([15])$. Let $\gamma$ be a closure operator on a residuated pogroupoid $\mathbf{G}$. Then $\gamma$ is a nucleus if and only if $x / y, y \backslash x \in G_{\gamma}$ for all $x \in G_{\gamma}, y \in G$.

Let $\mathbf{G}=(G, \leq, \cdot)$ be a residuated groupoid, $\gamma$ a nucleus on $\mathbf{G}$, and for all $x, y \in G$ define $x \circ_{\gamma} y=\gamma(x \circ y)$. $\mathbf{G}_{\gamma}=\left(G_{\gamma}, \leq, \circ_{\gamma}\right)$, is called the $\gamma$-image of $\mathbf{G}$. If $\mathbf{G}$ has a unit, is lattice ordered and/or is residuated, then the $\gamma$-retraction is defined to have the operations $\gamma(1), \wedge, \vee_{\gamma}$ (where $\left.x \vee_{\gamma} y=\gamma(x \vee y)\right)$, and $\backslash$, /, respectively.

Lemma 1.4 ([14, 12]).

(i) The nucleus retraction $\mathbf{G}_{\gamma}$ of a pogroupoid $\mathbf{G}$ is a pogroupoid and the properties of lattice-ordering, being residuated and having a unit are preserved.

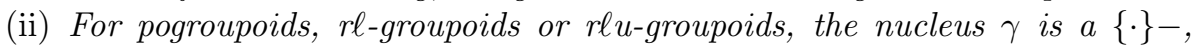
$\{\cdot, \vee\}-$ or $\{\cdot, \vee, 1\}$-homomorphism from $\mathbf{G}$ to $\mathbf{G}_{\gamma}$ respectively. In particular, if $t$ is $a\{\cdot, \vee, 1\}$-formula, then $\gamma\left(t^{\mathbf{G}}(\bar{x})\right)=t^{\mathbf{G}_{\gamma}}(\gamma(\bar{x}))$ for all sequences $\bar{x}$ of elements in $G$.

(iii) All equations and inequations involving $\{\cdot, \vee, 1\}$ are preserved. For example, if $\mathbf{G}$ is associative, commutative, integral or contracting, then so is $\mathbf{G}_{\gamma}$.

(iv) In particular, if $\mathbf{G}$ is a residuated lattice and $\gamma$ is a nucleus on it, then the $\gamma$-retraction $\mathbf{G}_{\gamma}$ of $\mathbf{G}$ is a residuated lattice.

Nuclei on powersets. A ternary relational structure is a pair $\mathbf{W}=(W, \circ)$, where $W$ is a set and $\circ \subseteq W^{3}$. On the powerset $\mathcal{P}(W)$ of $W$ we define the operation $X \circ Y=\{z \in W:(x, y, z) \in \circ$ for some $x \in X, y \in Y\}$ and we write $x \circ y$ for the set $\{x\} \circ\{y\}$ and $x \circ Y$ for $\{x\} \circ Y$. Also, we define the sets $X / Y=$ $\{z \mid\{z\} \circ Y \subseteq X\}$ and $Y \backslash X=\{z \mid Y \circ\{z\} \subseteq X\}$. It is easy to see that the algebra $\mathcal{P}(\mathbf{W})=(\mathcal{P}(W), \cap, \cup, \cdot, \backslash, /)$ is a residuated groupoid.

Lemma 1.5. Let $(W, \circ)$ be a ternary relation structure and let $\gamma$ be a closure operator on $\mathcal{P}(W)$ with basis $\mathcal{D}$. Then the following statements are equivalent:

(i) $\gamma$ is a nucleus on $\mathcal{P}(\mathbf{W})$.

(ii) $C /\{w\},\{w\} \backslash C \in \mathcal{P}(W)_{\gamma}$, for all $C \in \mathcal{D}$ and $w \in W$.

Proof. We use the equivalent condition for a nucleus given in Lemma 1.3, which obviously implies (ii). Conversely, assume (ii) holds, let $\mathbf{L}=\mathcal{P}(\mathbf{W})$, and consider $X \in L_{\gamma}$ and $Y \in L$. Since $\mathcal{D}$ is a basis for $\gamma$, there exists $\mathcal{X} \subseteq \mathcal{D}$ such that $X=\bigcap \mathcal{X}$. By Lemma 1.1. $X / Y=(\bigcap \mathcal{X}) /\left(\bigcup_{y \in Y}\{y\}\right)=\bigcap_{C \in \mathcal{X}} \bigcap_{y \in Y} C /\{y\}$. By assumption, $C /\{y\}$ is a $\gamma$-closed element; hence $X / Y$ is $\gamma$-closed since the intersection of closed elements of a closure operator on a complete lattice is also closed.

\section{Residuated frames And Gentzen fRAmes}

After giving the definition of a residuated frame, we discuss a range of examples that will play a role in the subsequent applications. For a ternary relational structure $(W, \circ)$, the condition below characterizes the relations $R \subseteq W \times W^{\prime}$ for which 
$\gamma_{R}$ is a nucleus on $\mathcal{P}(W, \circ)$. A relation $N \subseteq W \times W^{\prime}$ is called nuclear on $(W, \circ)$ if there exist ternary relations $\| \subseteq W \times W^{\prime} \times W^{\prime}$ and $\| \subseteq W^{\prime} \times W \times W^{\prime}$ such that for all $u, v \in W, w \in W^{\prime}$,

$$
u \circ v N w \text { iff } \quad v N u \| w \text { iff } \quad u N w / / v \text {. }
$$

This condition makes use of the notation $x N Y$ and $X N y$ defined earlier, as well as the notation $x \star y=\{z:(x, y, z) \in \star\}$ for $\star \in\{0, \mathbb{\|}, / /\}$.

Lemma 2.1. If $(W, \circ)$ is a ternary relation structure and $N \subseteq W \times W^{\prime}$, then $\gamma_{N}$ is a nucleus on $\mathcal{P}(W, \circ)$ iff $N$ is a nuclear relation.

Proof. By Lemma 1.2(iii), the collection $\mathcal{D}=\left\{\{w\}^{\triangleleft}: w \in W^{\prime}\right\}$ forms a basis for $\gamma_{N}$. So, by Lemma 1.3. $\gamma_{N}$ is a nucleus iff $\{w\}^{\triangleleft} /\{u\}$ and $\{u\} \backslash\{w\}^{\triangleleft}$ are $\gamma_{N^{-}}$ closed, for all $u \in W$ and $w \in W^{\prime}$ (here $\backslash$ and / are calculated in $\mathcal{P}(W, \circ)$ ). Since $\mathcal{D}$ is a basis, $\{u\} \backslash\{w\}^{\triangleleft}$ is closed iff $\{u\} \backslash\{w\}^{\triangleleft}=\bigcap \mathcal{X}$, for some $\mathcal{X} \subseteq \mathcal{D}$ iff $\{u\} \backslash\{w\}^{\triangleleft}=\bigcap_{c \in u \Downarrow w}\{c\}^{\triangleleft}$, for some $u \Downarrow w \subseteq W^{\prime}$. This is equivalent to the statement that for all $v \in W$,

$$
v \in\{u\} \backslash\{w\}^{\triangleleft} \quad \text { iff } \quad v \in \bigcap_{c \in u \backslash w}\{c\}^{\triangleleft} .
$$

Transforming this statement further we obtain for all $v \in W$,

$$
u \circ v \subseteq\{w\}^{\triangleleft} \quad \text { iff } \quad v \in\{c\}^{\triangleleft} \text { for all } c \in u \Downarrow w,
$$

or, equivalently, $u \circ v N w$ iff $v N c$ for all $c \in u \| w$. So, $\{u\} \backslash\{w\} \triangleleft$ is closed iff there exists $u \| w \subseteq W^{\prime}$ such that $u \circ v N w$ iff $v N u \| w$. Likewise, we obtain the second equivalence of a nuclear relation.

A residuated frame is a structure of the form $\mathbf{W}=\left(W, W^{\prime}, N, \circ, \|, / /\right)$, where $(W, \circ)$ is a ternary relational structure and $N \subseteq W \times W^{\prime}$ is a nuclear relation on $(W, \circ)$ with respect to $\mathbb{\|}, / /$. Concretely, this means

- $N$ is a binary relation from $W$ to $W^{\prime}$, called the Galois relation,

- $\circ \subseteq W^{3}, \quad\left\|\subseteq W \times W^{\prime} \times W^{\prime}, \quad\right\| \subseteq W^{\prime} \times W \times W^{\prime}$, and

- $(u \circ v) N w$ iff $v N(u \| w)$ iff $u N(w / / v)$ for all $u, v \in W$ and $w \in W^{\prime}$.

It follows from Lemma 1.4 and Lemma 2.1 that $\mathcal{P}(W, \circ)_{\gamma_{N}}$ is an $r \ell$-groupoid, called the Galois algebra of $\mathbf{W}$ and denoted by $\mathbf{W}^{+}$. In detail, $\mathbf{W}^{+}=\left(\gamma_{N}[\mathcal{P}(W)], \cap, \cup_{\gamma_{N}}\right.$, $\left.\circ_{\gamma_{N}}, \backslash, /\right)$, where

$$
\begin{array}{ll}
X \cup_{\gamma_{N}} Y=\gamma_{N}(X \cup Y), & X \backslash Y=\{z: X \circ z \subseteq Y\}, \quad Y / X=\{z: z \circ X \subseteq Y\}, \\
X \circ \gamma_{N} Y=\gamma_{N}(X \circ Y), & X \circ Y=\{z \in W: \exists x \in X, y \in Y((x, y, z) \in \circ)\} .
\end{array}
$$

A unital residuated frame (or $r u$-frame) $\mathbf{W}=\left(W, W^{\prime}, N, \circ,\|\|, E,\right)$ is a residuated frame with a set $E \subseteq W$ such that $(x \circ E)^{\triangleright}=\{x\}^{\triangleright}=(E \circ x)^{\triangleright}$, for all $x \in W$, and an ruz-frame $\mathbf{W}=\left(W, W^{\prime}, N, \circ, \|, / /, E, D\right)$ is an ru-frame with a distinguished subset $D \subseteq W$. In either case $\mathbf{W}^{+}$has a unit $1=\gamma_{N}(E)$, and in the latter case also has $\gamma_{N}(D)$ as the interpretation for the constant 0. A residuated frame is associative if $[(x \circ y) \circ z]^{\triangleright}=[x \circ(y \circ z)]^{\triangleright}$ for all $x, y, z \in W$. It is straightforward to check that the Galois algebra of an associative $r u$-frame is a residuated lattice, and the Galois algebra of an associative ruz-frame is an FL-algebra.

The residuated frames we consider extend the formal contexts of formal concept analysis [16. These structures consist of triples $\left(W, W^{\prime}, N\right)$, where $N \subseteq W \times W^{\prime}$, so they capture the lattice operations, but not the multiplication, residuals and unit element. Furthermore, residuated frames are related to, but more general than, the 
reduced separated frames (or RS-frames) of [17. The latter assume that the map $w \mapsto\{w\}^{\triangleright \triangleleft}$ is a bijection (i.e., the frame is separated) and that the copy of $W$ in $W^{+}$consists of completely join irreducible elements (i.e., the frame is reduced). These conditions make the relation $N$ behave like a partial order, the restriction of the order of $W^{+}$on the copy, in $W^{+}$, of the union $W \cup W^{\prime}$, and set up a duality between perfect lattices and RS-frames. The analogue of the ternary relation $\circ$ on $W$ in the context of RS-frames is given by a ternary relation $R \subseteq W \times W \times W^{\prime}$ that satisfies a compatibility condition. (Although for the applications that we consider in this paper $\circ$ will be a binary operation, our general framework applies to the more general case, where $\circ$ is a ternary relation and therefore connects to the study of RS-frames.) For RS-frames, the relations $\circ$ and $R$ are interdefinable by means of the nuclear relation $N$. In particular, the residuated frames that are needed for applications in this paper are rarely RS-frames. To illustrate the generality of residuated frames, we now consider a series of examples.

The Dedekind-MacNeille completion. Given a poset $\mathbf{P}=(P, \leq)$, we can define the residuated frame $\mathbf{W}_{\mathbf{P}}=(P, P, \leq, \circ, \mathbb{\|}, / /)$, where $\circ, \mathbb{\|}, / /$ are the empty set. The nuclear property for $\leq$ is vacuously true.

The poset $\mathcal{P}(P)_{\gamma_{\leq}}$(the poset reduct of $\mathbf{W}_{\mathbf{P}}^{+}$) of closed sets is called the DedekindMacNeille completion of $\mathbf{P}$. It is well known that the map $x \mapsto\{x\} \triangleleft$ is an embedding of $\mathbf{P}$ into $\mathbf{W}_{\mathbf{P}}^{+}$. More generally, let $\mathbf{G}=(G, \leq, \cdot, \backslash, /)$ be a residuated pogroupoid and define $x \circ y=\{x y\}, x \| y=\{x \backslash y\}$ and $x / / y=\{x / y\}$. Then $\mathbf{W}_{\mathbf{G}}=(G, G, \leq, \cdot, \|, / /)$ is a residuated frame since the nuclear property for $\leq$ is just the residuation property for $\mathbf{G}$. If $\mathbf{G}$ is associative or has a unit, then $\mathbf{W}_{\mathbf{G}}^{+}$ has the same properties. In particular, if $\mathbf{G}$ is a residuated lattice, then $\mathbf{W}_{\mathbf{G}}^{+}$is a complete residuated lattice, and the map $x \mapsto\{x\}^{\triangleleft}$ is an embedding of $\mathbf{G}$ into $\mathbf{W}_{\mathbf{G}}^{+}$(Corollary 2.9). Hence $\mathbf{W}_{\mathbf{G}}^{+}$is called the Dedekind-MacNeille completion of G.

Partial subalgebras. Let $\mathbf{A}$ be a residuated lattice and $\mathbf{B}$ a partial subalgebra of $\mathbf{A}$; i.e., $B$ is any subset of $A$, and each operation $f^{\mathbf{A}}$ on $A$ induces a partial operation $f^{\mathbf{B}}$ on $B$ by $f^{\mathbf{B}}\left(b_{1}, \ldots, b_{n}\right)=f^{\mathbf{A}}\left(b_{1}, \ldots, b_{n}\right)$ if this latter value is in $B$, and undefined otherwise. Define $(W, \circ, 1)$ to be the submonoid of $\mathbf{A}$ generated by B. A unary linear polynomial of $(W, \circ, 1)$ is a map $u$ on $W$ of the form $u(x)=$ $v \circ x \circ w$, for $v, w \in W$. Such polynomials are also known as sections and we denote the set of all sections by $S_{W}$. Let $W^{\prime}=S_{W} \times B$, and define $x N(u, b)$ by $u(x) \leq^{\mathbf{A}} b$. Given $y \in W$ and $u \in S_{W}$, define sections $u^{\prime}(x)=u(x \circ y)$ and $u^{\prime \prime}(y)=u(x \circ y)$. We will also use the notation $u^{\prime}=u\left(\bullet_{-} \circ y\right)$ and $u^{\prime \prime}=u\left(x \circ \circ_{-}\right)$. Now define $x \rrbracket(u, b)=\left\{\left(u\left(x \circ \circ_{-}\right), b\right)\right\}$ and $(u, b) / / y=\left\{\left(u\left({ }_{-} \circ y\right), b\right)\right\}$. Then it is easy to see that $\mathbf{W}_{\mathbf{A}, \mathbf{B}}=\left(W, W^{\prime}, N, \circ, \|, / /\right)$ is a residuated frame. This result also holds if $\mathbf{A}$ is just a pogroupoid (residuals are not needed in $\mathbf{A}$ ). Corollary 3.16 below shows that the map $b \mapsto\{(i d, b)\}^{\triangleleft}$ is an embedding of the partial subalgebra $\mathbf{B}$ of $\mathbf{A}$ into the $r \ell$-groupoid $\mathbf{W}_{\mathbf{A}, \mathbf{B}}^{+}$.

The system $G L$. Let $\mathcal{L}=\{\wedge, \vee, \cdot, \backslash, /, 1,0\}$ be the language of FL-algebras. Terms in this language correspond to propositional formulas in substructural logic; hence the set of all terms (over some fixed countable set of variables) is denoted by $\mathrm{Fm}$. Let $\circ$ be a binary symbol, $\varepsilon$ a constant symbol, and define $(W, \circ, \varepsilon)$ to be the free groupoid with unit $\varepsilon$ generated by the set $F m$. As in the partial subalgebra example, $S_{W}$ denotes the set of unary linear polynomials of $(W, \circ, \varepsilon)$. (However we 
TABLE 1. The system GL.

$$
\begin{gathered}
\frac{x \Rightarrow a \quad u(a) \Rightarrow c}{u(x) \Rightarrow c}(\mathrm{CUT}) \quad \frac{}{a \Rightarrow a}(\mathrm{Id}) \quad \frac{u(a \circ b) \Rightarrow c}{u(a \cdot b) \Rightarrow c}(\cdot \mathrm{L}) \quad \frac{x \Rightarrow a \quad y \Rightarrow b}{x \circ y \Rightarrow a \cdot b}(\cdot \mathrm{R}) \\
\frac{x \Rightarrow a \quad u(b) \Rightarrow c}{u(x \circ(a \backslash b)) \Rightarrow c}(\backslash \mathrm{L}) \quad \frac{a \circ x \Rightarrow b}{x \Rightarrow a \backslash b}(\backslash \mathrm{R}) \quad \frac{x \Rightarrow a \quad u(b) \Rightarrow c}{u((b / a) \circ x) \Rightarrow c}(/ \mathrm{L}) \quad \frac{x \circ a \Rightarrow b}{x \Rightarrow b / a}(/ \mathrm{R}) \\
\frac{u(a) \Rightarrow c}{u(a \wedge b) \Rightarrow c}(\wedge \mathrm{L} \ell) \quad \frac{u(b) \Rightarrow c}{u(a \wedge b) \Rightarrow c}(\wedge \mathrm{L} r) \quad \frac{x \Rightarrow a \quad x \Rightarrow b}{x \Rightarrow a \wedge b}(\wedge \mathrm{R}) \quad \frac{u(\varepsilon) \Rightarrow a}{u(1) \Rightarrow a}(1 \mathrm{~L}) \\
\frac{u(a) \Rightarrow c \quad u(b) \Rightarrow c}{u(a \vee b) \Rightarrow c}(\vee \mathrm{L}) \quad \frac{x \Rightarrow a}{x \Rightarrow a \vee b}(\vee \mathrm{R} \ell) \quad \frac{x \Rightarrow b}{x \Rightarrow a \vee b}(\vee \mathrm{R} r) \quad \frac{}{\varepsilon \Rightarrow 1}(1 \mathrm{R})
\end{gathered}
$$

do not assume associativity of $\circ$; hence $u(x)$ cannot in general be written in the form $v \circ x \circ w$.) A (single-conclusion) sequent is a pair $(x, b) \in W \times F m$, which is traditionally written $x \Rightarrow b$, and the symbol $\Rightarrow$ is called the sequent separator. A sequent rule is a pair $\left(\left\{s_{1}, \ldots, s_{n}\right\}, s_{0}\right)$ where $s_{0}, \ldots, s_{n}$ are sequents. Such rules are usually presented in the form

$$
\begin{array}{llll}
s_{1} & s_{2} & \ldots & s_{n} \\
s_{0} & \text { or } & - \\
s_{0}
\end{array}
$$

with rules of the latter form referred to as axioms. Finally, a Gentzen system is a set of sequent rules.

Consider the Gentzen system GL for the nonassociative full Lambek calculus, given by the rules in Table 1 and all their uniform substitution instances (i.e., $a, b, c$ range over $F m, x, y$ range over $W$ and $u$ ranges over $S_{W}$ ). The system is essentially obtained from Gentzen's system LJ for intuitionistic logic, by removing all the implicit structural rules. A proof in GL is defined inductively in the usual way as a labeled rooted tree (where the order of the branches does not matter). Formally, every rule $(S, s)$ is considered as a proof with assumption $S$ and conclusion s. Moreover, if $\mathrm{P}_{1}, \ldots, \mathrm{P}_{n}$ are proofs with sets of assumptions $S_{1}, \ldots, S_{n}$ and conclusions $s_{1}, \ldots, s_{n}$, respectively, and if $\left(\left\{s_{1}, \ldots s_{n}\right\}, s_{0}\right)$ is an instance of a rule in GL, then the tree (denoted by)

$$
\frac{\mathrm{P}_{1} \quad \ldots \quad \mathrm{P}_{n}}{s_{0}}
$$

is a proof with set of assumptions $S_{1} \cup \cdots \cup S_{n}$ and conclusion $s_{0}$. If there is a proof of a sequent $s$ in GL from assumptions $S$, then we write $S \vdash_{\mathbf{G L}} s$ and say that $s$ is provable in GL from $S$. If $S$ is empty we simply write $\vdash_{\mathbf{G L}} s$ and say that $s$ is provable in GL. For more on GL, see [14].

Now take $W^{\prime}=S_{W} \times F m$, where $S_{W}$ is the set of all unary linear polynomials in $W$ and define the relation $N$ by

$$
x N(u, a) \quad \text { iff } \quad \vdash_{\mathbf{G L}}(u(x) \Rightarrow a) .
$$

Then $x \circ y N(u, a)$ iff $\vdash_{\mathbf{G L}} u(x \circ y) \Rightarrow a$ iff $x N\left(u\left({ }_{-} \circ y\right), a\right)$ iff $y N\left(u\left(x \circ{ }_{-}\right), a\right)$. Hence $N$ is a nuclear relation, where the appropriate subsets of $W^{\prime}$ are given by

$$
(u, a) / / x=\left\{\left(u\left(\__{-} \circ x\right), a\right)\right\} \text { and } x \rrbracket(u, a)=\left\{\left(u\left(x \circ_{-}\right), a\right)\right\} .
$$

The resulting residuated frame is denoted by $\mathbf{W}_{\mathbf{G L}}$. Let $\mathbf{F m}$ be the countably generated absolutely free algebra over the language of FL-algebras. Unlike some of 
the previous examples we cannot expect the map $a \mapsto\{(i d, a)\} \triangleleft$ to be an embedding of $\mathbf{F m}$ into $\mathbf{W}_{\mathbf{G L}}^{+}$. However we will show that this map has some weak properties of a homomorphism, referred to as a quasi-homomorphism in [2].

We will use this quasi-homomorphism to prove the cut-elimination property for GL, namely that the system obtained from GL by removing (all instances of) the cut rule has exactly the same provable sequents as GL.

We say that an $r \ell u$-groupoid $\mathbf{G}$ satisfies the sequent $x \Rightarrow a$ (also that the sequent holds or is valid in $\mathbf{G}$ ) if for every homomorphism $f: \mathbf{F m} \rightarrow \mathbf{G}, f\left(x^{\mathbf{F m}}\right) \leq f(a)$. Here $x^{\mathbf{F m}}$ denotes the formula obtained from $x$ by replacing $\circ$ with $\cdot ;$ also $\varepsilon^{\mathbf{F m}}=1$. The following well-known result states that GL is sound with respect to all $r \ell u$ groupoids. The proof proceeds by induction on the rules (and axioms) of GL.

Lemma 2.2 (Soundness). Every sequent that is provable in $\mathbf{G L}$ is valid in all rlu-groupoids.

The converse is also true, i.e., $r \ell$-groupoids provide a complete semantics. (This is also well known; see Theorem 3.2 and Corollary 3.3 below for an algebraic proof.) The system $\mathbf{G L}_{\mathbf{a}}$ is defined to be $\mathbf{G L}$ augmented by the structural rule of associativity

$$
\frac{u((x \circ y) \circ z) \Rightarrow c}{\overline{u(x \circ(y \circ z)) \Rightarrow c}}(\mathrm{a}) .
$$

The double line means that we assume two rules, the one stated (read downward) and its inverse (read upward). Other structural rules can be added to obtain further basic substructural logic systems, exchange, contraction, left weakening (or integrality) and right weakening

$$
\frac{u(x \circ y) \Rightarrow c}{u(y \circ x) \Rightarrow c} \text { (e) } \quad \frac{u(x \circ x) \Rightarrow c}{u(x) \Rightarrow c} \text { (c) } \quad \frac{u(\varepsilon) \Rightarrow c}{u(x) \Rightarrow c} \text { (i) } \quad \frac{x \Rightarrow \varepsilon}{x \Rightarrow c} \text { (o). }
$$

The (o) rule is only effective in an extension $\mathbf{G L}^{0}$ of $\mathbf{G L}$ with the rules

$$
\frac{x \Rightarrow \varepsilon}{x \Rightarrow 0}(0 \mathrm{R}) \quad \overline{0 \Rightarrow \varepsilon}(0 \mathrm{~L})
$$

where the right-hand sides of sequents in $\mathbf{G L}^{0}$ are allowed to be $\varepsilon$. The sequent $x \Rightarrow \varepsilon$ is valid in an ruz-groupoid $\mathbf{G}$ if for every homomorphism $f: \mathbf{F m} \rightarrow \mathbf{G}$, $f\left(x^{\mathbf{F m}}\right) \leq 0$. Note that a residuated frame for the system $\mathbf{G L}^{0}$ uses $W^{\prime}=S_{W} \times$ $(F m \cup\{\varepsilon\})$. It is easy to see that the sequent $x \Rightarrow \varepsilon$ is provable in $\mathbf{G L}^{0}$ iff the sequent $x \Rightarrow 0$ is provable. Also, the systems $\mathbf{G L}$ and $\mathbf{G} \mathbf{L}^{0}$ prove the same sequents with nonempty right-hand side. In that sense the two systems are essentially equivalent, but $\mathbf{G L}^{0}$ supports the addition of further structural rules, like (o). We extend the subscript notation, so for example $\mathbf{G L}_{\mathbf{a e}}$ is $\mathbf{G L}$ plus associativity and exchange. Furthermore we abbreviate the combination of (i) and (o) to weakening (w). The system $\mathbf{G L}_{\mathbf{a e c w}}^{\mathbf{0}}$ is equivalent to Gentzen's original system $\mathbf{L J}$ for intuitionistic logic.

The system $F L$. The Gentzen system $\mathbf{F L}$ is an associative version of $\mathbf{G L}^{0}$. The only difference is that now $W$ (containing the left-hand sides of sequents) is defined as the free monoid over the set $F m$ of formulas. Consequently $x, y, z$ range over sequences of formulas. Note that $\mathbf{F L}$ has the same rules as $\mathbf{G L}^{0}$, but different rule instances. Traditionally sequents such as $u(a) \Rightarrow c$ are denoted by $\Gamma, A, \Delta \Rightarrow C$. The system FL was introduced by $\mathrm{H}$. Ono and is called full Lambek calculus. In contrast, Lambek calculus is a system without connectives and rules for $\wedge, \vee, 1,0$. 
Since $\circ$ is an associative operation, we omit any parentheses. In fact $\circ$ is traditionally denoted by a comma, and the elements of $W$ are concretely realized as finite sequences of formulas. On the other hand, the operation · on $\mathbf{F m}$ is not associative: from the sequent $a \circ b \circ c \Rightarrow d$ one can prove the distinct sequents $(a \cdot b) \cdot c \Rightarrow d$ and $a \cdot(b \cdot c) \Rightarrow d$ by using two applications of $(\cdot \mathrm{L})$. Note that FL is equivalent to the system $\mathbf{G L}_{\mathbf{a}}^{0}$. Likewise, $\mathbf{F L}_{\mathbf{e}}$ is equivalent to $\mathbf{G L}_{\mathbf{a e}}^{0}$. As shown by the next well-known soundness result, the naming similarity between $\mathbf{F L}$ and FL-algebras is not a coincidence.

Lemma 2.3. Every sequent that is provable in $\mathbf{F L}$ is valid in all FL-algebras.

It turns out that FL is an equivalent algebraic semantics for FL. For more on FL, see for example [12. The corresponding residuated frame $\mathbf{W}_{\mathbf{F L}}$ is associative.

Remark 2.4. The systems PL and $\mathbf{M L}$, considered in the literature, are covered by our analysis; see e.g. [14 for the definitions. Although the verification is not trivial, we omit any relevant discussion due to space limitations.

Gentzen frames. After a short discussion of the similarities of the previous examples, we define a common abstraction called a Gentzen frame. This allows us to prove a quasi-homomorphism result that yields simultaneously the three embeddings claimed in the last section and will be instrumental in obtaining the new results in the paper. Note that in $\mathbf{W}_{\mathbf{G L}}$, if $a N(u, c)$ and $b N(u, c)$, then $\vdash_{\mathbf{G L}} u(a) \Rightarrow c$ and $\vdash_{\mathbf{G L}} u(b) \Rightarrow c$. In view of the rule $(\vee \mathrm{L})$, we obtain $\vdash_{\mathbf{G L}} u(a \vee b) \Rightarrow c$, namely $a \vee b N(u, c)$. Hence in $\mathbf{W}_{\mathbf{G L}}$ we have the implication

$$
\text { if } a N z \text { and } b N z \text {, then } a \vee b N z \text {. }
$$

The same argument works for $\mathbf{W}_{\mathbf{F L}}$. Interestingly enough, the same implication holds for $\mathbf{W}_{\mathbf{G}}$, where $\mathbf{G}$ is a $G L$-algebra, i.e., an rluz-groupoid. Indeed, if a $N c$ and $b N c$, then $a \leq c$ and $b \leq c$, so $a \vee b \leq c$ and $a \vee b N c$. Furthermore, if $\mathbf{A}$ is an $r \ell u$-groupoid, $\mathbf{B}$ a partial subalgebra of $\mathbf{A}$ and $a, b, a \vee b \in B$, then $a N c$ and $b N c$; namely $a \leq c$ and $b \leq c$ implies $a \vee b \leq c$ and $a \vee b N c$. In other words, the above implication, also written in the form

$$
\frac{a N z b N z}{a \vee b N z}(\vee \mathrm{L})
$$

holds in the residuated frames $\mathbf{W}_{\mathbf{G} \mathbf{L}}, \mathbf{W}_{\mathbf{F L}}, \mathbf{W}_{\mathbf{G}}, \mathbf{W}_{\mathbf{A}, \mathbf{B}}$, for all $z \in W^{\prime}$ and all $a, b$ that are elements of $F m$ for the first two frames, elements of $G$ for $\mathbf{W}_{\mathbf{G}}$, and elements of $B$ for $\mathbf{W}_{\mathbf{A}, \mathbf{B}}$. Note that the sets $F m, G$ and $B$ are subsets of $W$, in the corresponding frames, and they actually generate it as a groupoid under the operation $\circ$. Moreover, they are all (partial) $\mathcal{L}$-algebras. Furthermore, these sets can be identified with a subset of $W^{\prime}$ and their elements are exactly the right-hand sides of the sequents in each case. In the case of $\mathbf{W}_{\mathbf{G L}}$ and $\mathbf{W}_{\mathbf{F L}}$, every $b \in F m$ can be identified with the element $(i d, b)$ of $W^{\prime}$, where $i d$ is the identity polynomial. The same identification works for elements of $B$ for $\mathbf{W}_{\mathbf{A}, \mathbf{B}}$. Finally, in the case of $\mathbf{W}_{\mathbf{G}}, W^{\prime}=G$ itself. These considerations lead to the following definition about a pair of a residuated frame and a special partial $\mathcal{L}$-algebra.

A Gentzen ru-frame (or simply Gentzen frame) is a pair $(\mathbf{W}, \mathbf{B})$, where

(i) $\mathbf{W}=\left(W, W^{\prime}, N, \circ, \mathbb{\|}, \mathbb{\|},\{\varepsilon\}\right)$ is an $r u$-frame with $\circ$ a binary operation,

(ii) $\mathbf{B}$ is a partial $\mathcal{L}$-algebra,

(iii) $(W, \circ, \varepsilon)$ is a groupoid with unit generated by $B \subseteq W$, 
TABLE 2. The theory GN.

$$
\begin{aligned}
& \frac{x N a \quad a N z}{x N z}[\mathrm{CUT}] \quad \overline{a N a}[\mathrm{Id}] \quad \frac{a \circ b N z}{a \cdot b N z}[\cdot \mathrm{L}] \quad \frac{x N a y N b}{x \circ y N a \cdot b}[\cdot \mathrm{R}] \\
& \frac{x N a b N z}{x \circ(a \backslash b) N z}[\backslash \mathrm{L}] \quad \frac{a \circ x N b}{x N a \backslash b}[\backslash \mathrm{R}] \quad \frac{x N a b N z}{(b / a) \circ x N z}[/ \mathrm{L}] \quad \frac{x \circ a N b}{x N b / a}[/ \mathrm{R}] \\
& \frac{a N z}{a \wedge b N z}[\wedge \mathrm{L} \ell] \quad \frac{b N z}{a \wedge b N z}[\wedge \mathrm{L} r] \quad \frac{x N a x N b}{x N a \wedge b}[\wedge \mathrm{R}] \quad \frac{\varepsilon N z}{1 N z}[1 \mathrm{~L}] \\
& \frac{a N z b N z}{a \vee b N z}[\mathrm{VL}] \quad \frac{x N a}{x N a \vee b}[\mathrm{VR} \ell] \quad \frac{x N b}{x N a \vee b}[\mathrm{VR} r] \quad \overline{\varepsilon N 1}[1 \mathrm{R}]
\end{aligned}
$$

(iv) there is an injection of $B$ into $W^{\prime}$ (under which we will identify $B$ with a subset of $W^{\prime}$ ) and

(v) $N$ satisfies the rules of GN (Table 2) for all $a, b \in B, x, y \in W$ and $z \in W^{\prime}$.

A rule is understood to hold only in case all the expressions in it make sense. For example, $(\wedge \mathrm{L} \ell)$ is read as, if $a, b, a \wedge b \in B, z \in W^{\prime}$ and $a N z$, then $a \wedge b N z$.

A Gentzen ruz-frame is an expansion of a Gentzen $r u$-frame such that $\mathbf{W}$ contains an extra constant, evaluated as $\{\varepsilon\}^{\triangleleft}$, and (iv),(v) are modified as follows:

(iv') there is an injection of $B \cup\{\varepsilon\}$ into $W^{\prime}$ (under which we will identify $B \cup\{\varepsilon\}$ with a subset of $\left.W^{\prime}\right)$ and

$\left(\mathrm{v}^{\prime}\right) N$ satisfies the rules of GN (Table 2) for all $a, b \in B, x, y \in W$ and $z \in W^{\prime}$ as well as

$$
\frac{x N \varepsilon}{x N 0}[0 \mathrm{R}] \quad \overline{0 N \varepsilon}[0 \mathrm{~L}] .
$$

A cut-free Gentzen frame is defined in the same way, but it is not stipulated to satisfy the [CUT] rule. Gentzen frames are a proper generalization of Gentzen matrices, considered in [14; the latter are special cases where $W^{\prime}=S_{W} \times B$. However, there are applications in the forthcoming work of the first author and K. Terui, where for example $W^{\prime}=S_{W} \times B^{2}$. A sequent in the (possibly cut-free) Gentzen frame $(\mathbf{W}, \mathbf{B})$ is an element of $W \times B$. We use the notation $x \Rightarrow a$, or $x \leq a$ for such a pair. The verification of the following result is straightforward.

Lemma 2.5. Using the notation of the previous section, $\left(\mathbf{W}_{\mathbf{G}}, \mathbf{G}\right),\left(\mathbf{W}_{\mathbf{A}, \mathbf{B}}, \mathbf{B}\right)$, $\left(\mathbf{W}_{\mathbf{G L}}, \mathbf{F m}\right)$ and $\left(\mathbf{W}_{\mathbf{F L}}, \mathbf{F m}\right)$ are Gentzen frames.

We will see more Gentzen frames later. The following theorem yields a common generalization of the embeddings of the previous section.

Theorem 2.6. Let $(\mathbf{W}, \mathbf{B})$ be a cut-free Gentzen ru-frame. For all $a, b \in B$, $X, Y \in \mathbf{W}^{+}$and for every connective $\bullet$, if $a \bullet^{\mathbf{B}} b$ is defined, then

(i) $1^{\mathbf{B}} \in \gamma_{N}(\{\varepsilon\}) \subseteq\left\{1^{\mathbf{B}}\right\}^{\triangleleft}$.

(ii) If $a \in X \subseteq\{a\}^{\triangleleft}$ and $b \in Y \subseteq\{b\}^{\triangleleft}$, then $a \bullet^{\mathbf{B}} b \in X \bullet^{\mathbf{W}^{+}} Y \subseteq\left\{a \bullet^{\mathbf{B}} b\right\}^{\triangleleft}$.

(iii) In particular, $a \bullet^{\mathbf{B}} b \in\{a\}^{\triangleleft} \bullet^{\mathbf{W}^{+}}\{b\}^{\triangleleft} \subseteq\left\{a \bullet^{\mathbf{B}} b\right\}^{\triangleleft}$.

(iv) If, additionally, $N$ satisfies [CUT], then $\{a\}^{\triangleleft} \bullet \mathbf{W}^{+}\{b\}^{\triangleleft}=\left\{a \bullet \bullet^{\mathbf{B}} b\right\}^{\triangleleft}$.

Furthermore, if $(\mathbf{W}, \mathbf{B})$ is a cut-free ruz-frame we have

(v) $0^{\mathbf{B}} \in\{\varepsilon\}^{\triangleleft} \subseteq\left\{0^{\mathbf{B}}\right\}^{\triangleleft}$. 
Proof. (i) Here $\bullet=1$, so by assumption $1^{\mathbf{B}}$ is defined. By [1R], we have $\varepsilon \in\left\{1^{\mathbf{B}}\right\}^{\triangleleft}$, so $\gamma_{N}(\{\varepsilon\}) \subseteq\left\{1^{\mathbf{B}}\right\}^{\triangleleft}$. On the other hand, if $\gamma_{N}(\{\varepsilon\}) \subseteq\{z\}^{\triangleleft}$, then $\varepsilon \in\{z\}^{\triangleleft}$ and $\varepsilon N z$. Therefore $1^{\mathbf{B}} N z$ by [1L], and hence $1^{\mathbf{B}} \in\{z\}^{\triangleleft}$. Thus, $1^{\mathbf{B}} \in \gamma_{N}(\{\varepsilon\})$.

(ii) We will give the proof for the connectives $\vee$, a and $\backslash$. The proof for the remaining two connectives follows the same ideas.

Let $\bullet=\vee$. If $x \in X$, then $x \in\{a\}^{\triangleleft}$, or equivalently $x N a$. By [VR $\ell$ ], $x N a \vee b$; hence $x \in\{a \vee b\}^{\triangleleft}$. Consequently $X \subseteq\{a \vee b\}^{\triangleleft}$, and similarly,

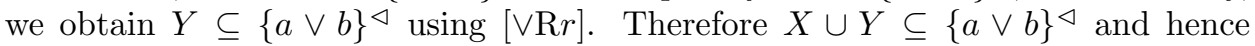
$X \vee Y=\gamma_{N}(X \cup Y) \subseteq\{a \vee b\}^{\triangleleft}$. On the other hand, let $z \in W^{\prime}$ and assume $X \vee Y \subseteq\{z\}^{\triangleleft}$. Then $a \in X \subseteq X \vee Y \subseteq\{z\}^{\triangleleft}$, so $a N z$. Similarly, $b N z$, so $a \vee b N z$ by [VL]; hence $a \vee b \in\{z\} \triangleleft$. Thus, $a \vee b \in X \vee Y$, by Lemma 1.2(iv).

Let $\bullet=\cdot$. If $x \in X$ and $y \in Y$, then $x \in\{a\}^{\triangleleft}$ and $y \in\{b\}^{\triangleleft}$, i.e., $x N a$ and $y N$. It follows from [.R] that $x \circ y N a \cdot b$; hence $x \circ y \in\{a \cdot b\}^{\triangleleft}$. Consequently, $X \circ Y \subseteq\{a \cdot b\}^{\triangleleft}$ and therefore $X \cdot \mathbf{W}^{+} Y=\gamma_{N}(X \circ Y) \subseteq\{a \cdot b\}^{\triangleleft}$.

On the other hand, let $z \in W^{\prime}$ and assume $X \cdot \mathbf{W}^{+} Y \subseteq\{z\}^{\triangleleft}$. Since $a \circ b \in$ $X \circ Y \subseteq \gamma_{N}(X \circ Y)=X \cdot \mathbf{W}^{+} Y$, we have $a \circ b \in\{z\}^{\triangleleft}$, so $a \circ b N z$. Consequently, $a \cdot b N z$, by [.L]; hence $a \cdot b \in\{z\}^{\triangleleft}$. Thus, $a \cdot b \in X \cdot \mathbf{W}^{+} Y$.

Let $\bullet=\backslash$. If $x \in X \backslash \mathbf{W}^{+} Y$, then $X \circ\{x\} \subseteq Y$. Since $a \in X$ and $Y \subseteq\{b\}^{\triangleleft}$, we have $a \circ x \in\{b\}^{\triangleleft}$, i.e., $a \circ x N b$. By $[\backslash \mathrm{R}]$ we obtain $x N a \backslash b$; hence $x \in\{a \backslash b\}^{\triangleleft}$.

On the other hand, if $Y \subseteq\{z\}^{\triangleleft}$, then $b \in\{z\}^{\triangleleft}$, so $b N z$. For all $x \in\{a\}^{\triangleleft}$, $x N a$, so $x \circ(a \backslash b) N z$, by $[\backslash \mathrm{L}]$, i.e., $x \circ(a \backslash b) \in\{z\}^{\triangleleft}$, for all $x \in\{a\}^{\triangleleft}$. Consequently, $\{a\}^{\triangleleft} \circ\{a \backslash b\} \subseteq\{z\}^{\triangleleft}$, for all $\{z\}^{\triangleleft}$ that contain $Y$, so $\{a\}^{\triangleleft} \circ\{a \backslash b\} \subseteq Y$. Since $X \subseteq\{a\}^{\triangleleft}$, we have $X \circ\{a \backslash b\} \in Y$, so $a \backslash b \in X \backslash \mathbf{W}^{+} Y$.

Statement (iii) is a direct consequence of (ii) for $X=\{a\}^{\triangleleft}$ and $Y=\{b\}^{\triangleleft}$.

(iv) We first show that if [CUT] holds, $c \in Z \subseteq\{c\}^{\triangleleft}$ and $Z$ is closed, then $\{c\}^{\triangleleft}=Z$. If $x \in\{c\}^{\triangleleft}$, then $x N c$. To show that $x \in Z$, let $Z \subseteq\{z\}^{\triangleleft}$, for some $z \in W^{\prime}$. Since $c \in Z$ by assumption, we get $c \in\{z\}^{\triangleleft}$, or equivalently $c N z$. By [CUT] we obtain $x N z$, namely $x \in\{z\}^{\triangleleft}$. Hence $x \in Z$ by Lemma 1.2(iv). Taking $c=a \bullet b$ and $Z=\{a\}^{\triangleleft} \bullet \mathbf{W}^{+}\{b\}^{\triangleleft}$, we obtain $\{a\}^{\triangleleft} \bullet \mathbf{W}^{+}\{b\}^{\triangleleft}=\{a \bullet b\}^{\triangleleft}$ from (ii).

(v) The [0L] rule immediately implies that $0^{\mathbf{B}} \in\{\varepsilon\}^{\triangleleft}$, and it follows from [0R] that $x \in\{\varepsilon\}^{\triangleleft}$ implies $x \in\left\{0^{\mathbf{B}}\right\}^{\triangleleft}$ for any $x \in W$. Hence $\{\varepsilon\}^{\triangleleft} \subseteq\left\{0^{\mathbf{B}}\right\}^{\triangleleft}$.

Given the assumption that $N$ is nuclear, the conditions in GN are not only sufficient, but also necessary for condition (ii). Note that if the frame satisfies only some of the conditions of $\mathbf{G N}$, then we obtain a result only for the corresponding connectives. Also, the result applies to generalizations of the Gentzen frame, where $W$ is not necessarily a groupoid and $B$ is a relational structure.

Corollary 2.7. If $(\mathbf{W}, \mathbf{B})$ is a Gentzen frame, the map $x \mapsto\{x\}^{\triangleleft}$ from $\mathbf{B}$ to $\mathbf{W}^{+}$

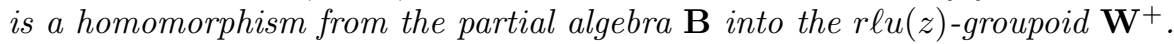

If $(\mathbf{W}, \mathbf{B})$ is a cut-free Gentzen frame, then $x \mapsto\{x\}^{\triangleleft}$ comes close to being a homomorphism. We refer to a map that satisfies condition (ii) of Theorem 2.6 as a quasi-homomorphism, and if it is 1-1 it is called a quasi-embedding.

In a cut-free Gentzen frame $(\mathbf{W}, \mathbf{B})$ the relation $N$ is called antisymmetric on $B$ if, for all $a, b \in B, a N b$ and $b N a$ implies $a=b$.

Corollary 2.8. If $(\mathbf{W}, \mathbf{B})$ is a (cut-free) Gentzen frame and $N$ is antisymmetric on $B$, then the map $x \mapsto\{x\} \triangleleft$ from $\mathbf{B}$ to $\mathbf{W}^{+}$is a (quasi-)embedding. 
Proof. We only need to show that the map in injective. Assume $\{a\}^{\triangleleft}=\{b\}^{\triangleleft}$, for $a, b \in B$. Recall that $a \in\{a\}^{\triangleleft}$ and $b \in\{b\}^{\triangleleft}$, so $a N b$ and $b N a$; hence $a=b$.

Embedding into the DM-completion and representation. Clearly if $\mathbf{G}$ is an $r \ell u$-groupoid, the Galois relation $\leq$ of $\mathbf{W}_{\mathbf{G}}$ is antisymmetric on $G$. The result below shows that every residuated lattice can be embedded into a complete one.

Corollary 2.9. Let $\mathbf{G}$ be an rlu-groupoid. The map $x \mapsto\{x\}^{\triangleleft}$ from $\mathbf{G}$ to $\mathbf{W}_{\mathbf{G}}^{+}$is an embedding.

Corollary 2.10 ([5]). Every residuated lattice is a subalgebra of the nucleus image of the powerset of a monoid.

Similarly it follows that every commutative residuated lattice is a subalgebra of a nucleus image of the powerset of a commutative monoid. More generally, since all monoid identities are preserved by nuclei, residuated lattices defined by monoid equations that are preserved under the powerset construction are obtained from the class of monoids that satisfy these identities. This includes all linear and balanced monoid identities (an identity is linear if each variable appears at most once on each side, and balanced if each variable appears on both sides).

\section{Cut elimination, FMP and FEP}

The cut-elimination property for a Gentzen system states that the set of provable sequents does not change if the cut rule is removed from the system. Since the effect of the cut rule cannot be simulated by composing the other rules (cut is not derivable) traditional arguments rewrite the proof, using multiple induction (on a complexity measure) and case analysis, by pushing instances of the cut rule upward.

A semantical proof of cut elimination for $\mathbf{F L}$ was given in [2] and later extended in 14] to $\mathbf{G L}, \mathbf{F L}, \mathbf{F L}_{\mathbf{e c}}$ and various extensions. We will obtain cut elimination for all these systems as corollaries of a general theorem that has many more consequences 8], 9. Since the notion of a sequent differs in these systems, we prove the theorem in a general setting and then instantiate it to the particular cases.

Cut elimination. Let $(\mathbf{W}, \mathbf{B})$ be a cut-free Gentzen $r u(z)$-frame with the added assumption that $\mathbf{B}$ is a total $\mathcal{L}$-algebra. For every homomorphism $f: \mathbf{F m} \rightarrow \mathbf{B}$, we let $\bar{f}: \mathbf{F m} \rightarrow \mathbf{W}^{+}$be the $\mathcal{L}$-homomorphism that extends the assignment $p \mapsto$ $\{f(p)\}^{\triangleleft}$, for all variables $p$ of $\mathbf{F m}$. [More generally, we may define the assignment by $p \mapsto Q_{p}$, where $Q_{p}$ is any closed set such that $f(p) \in Q_{p} \subseteq\{f(p)\}^{\triangleleft}$.]

Lemma 3.1. If $(\mathbf{W}, \mathbf{B})$ is a cut-free Gentzen frame and $\mathbf{B}$ a total algebra, then for every homomorphism $f: \mathbf{F m} \rightarrow \mathbf{B}$, we have $f(a) \in \bar{f}(a) \subseteq\{f(a)\}^{\triangleleft}$, for all $a \in F m$. If $(\mathbf{W}, \mathbf{B})$ is a Gentzen frame, then $\bar{f}(a)=\{f(a)\} \triangleleft$, for all $a \in F m$.

Proof. Let $f: \mathbf{F m} \rightarrow \mathbf{B}$ be a homomorphism. By definition of $\bar{f}$ and the axiom [Id], the statement holds for the propositional variables. For $a=1$, by Theorem 2.6)(i), we have $f(1)=1^{\mathbf{B}} \in \gamma_{N}(\{\varepsilon\})=1^{\mathbf{W}^{+}} \subseteq\left\{1^{\mathbf{B}}\right\}^{\triangleleft}=\{f(1)\}^{\triangleleft}$. By way of induction, assume that $f(a) \in \bar{f}(a) \subseteq\{f(a)\}^{\triangleleft}$ and $f(b) \in \bar{f}(b) \subseteq\{f(b)\}^{\triangleleft}$. By Theorem 2.6(iii), for each connective $\bullet$, we have $f(a) \bullet B f(b) \in \bar{f}(a) \bullet \mathbf{W}^{+} \bar{f}(b) \subseteq$ $\{f(a) \bullet \mathbf{B} f(b)\} \triangleleft$. Since $f$ and $\bar{f}$ are homomorphisms, we have $f(a \bullet b) \in \bar{f}(a \bullet b) \subseteq$ $\{f(a \bullet b)\}^{\triangleleft}$. Finally, if $(\mathbf{W}, \mathbf{B})$ is a Gentzen frame, then $\bar{f}(a)=\{f(a)\}^{\triangleleft}$, by Theorem 2.6(iv). 
To account for the different types of sequents in the applications we will use the most general type. For this section an (intuitionistic) sequent is an element of $F m^{\circ} \times F m$, where $\mathbf{F m}^{\circ}=\left(F m^{\circ}, \circ, \varepsilon\right)$ denotes the absolutely free algebra in the signature $\{\circ, \varepsilon\}$ over the set $F m$. We use the notation $x \Rightarrow a$ for sequents.

Let $(\mathbf{W}, \mathbf{B})$ be a cut-free Gentzen frame. Note that every map $f: F m \rightarrow B$ extends inductively to a map $f^{\circ}: F^{\circ} \rightarrow W$ by $f^{\circ}\left(x \circ^{\mathbf{F m}^{\circ}} y\right)=f^{\circ}(x) \circ W f^{\circ}(y)$. Likewise, every homomorphism $f: \mathbf{F m} \rightarrow \mathbf{G}$ into an $\mathcal{L}$-algebra $\mathbf{G}$ extends to a homomorphism $f^{\circ}: \mathbf{F m}^{\circ} \rightarrow \mathbf{G}$. For a total algebra $\mathbf{B}$, a sequent $x \Rightarrow a$ is said to be valid in $(\mathbf{W}, \mathbf{B})$ if for every homomorphism $f: \mathbf{F m} \rightarrow \mathbf{B}$, we have $f^{\circ}(x) N f(a)$. A sequent $x \Rightarrow a$ is said to be valid in a residuated $\ell$-groupoid $\mathbf{G}$ if it is valid in the Gentzen frame $\left(\mathbf{W}_{\mathbf{G}}, \mathbf{G}\right)$, namely if for all homomorphisms $f: \mathbf{F m} \rightarrow \mathbf{G}$, we have $f^{\circ}(x) \leq f(a)$.

Theorem 3.2. If $(\mathbf{W}, \mathbf{B})$ is a cut-free Gentzen $r(u)(z)$-frame and $\mathbf{B}$ is a total algebra, then every sequent that is valid in $\mathbf{W}^{+}$is also valid in $(\mathbf{W}, \mathbf{B})$.

Proof. Assume that $x \Rightarrow a$ is valid in $\mathbf{W}^{+}$and let $f: \mathbf{F m} \rightarrow \mathbf{B}$ be a homomorphism. We will show that $f^{\circ}(x) N f(a)$. Since $x \Rightarrow a$ is valid in $\mathbf{W}^{+}$, for the homomorphism $\bar{f}: \mathbf{F m} \rightarrow \mathbf{W}^{+}$, we have $\bar{f}^{\circ}(x) \subseteq \bar{f}(a)$. If $x=t^{\mathbf{F m}}{ }^{\circ}\left(b_{1}, \ldots, b_{n}\right)$, for $b_{1}, \ldots, b_{n} \in F m$, then $\bar{f}^{\circ}(x)=\bar{f}^{\circ}\left(t^{\mathbf{F m}}{ }^{\circ}\left(b_{1}, \ldots, b_{n}\right)\right)=t^{\mathbf{W}^{+}}\left(\bar{f}\left(b_{1}\right), \ldots, \bar{f}\left(b_{n}\right)\right)$. By Lemma 3.1. $\bar{f}(a) \subseteq\{f(a)\}^{\triangleleft}$ and $f\left(b_{i}\right) \in \bar{f}\left(b_{i}\right)$, for $i=1, \ldots, n$. Hence

$$
\begin{aligned}
f^{\circ}(x) & =f^{\circ}\left(t^{\mathbf{F m}^{\circ}}\left(b_{1}, \ldots, b_{n}\right)\right) & & \\
& =t^{(W, \circ)}\left(f\left(b_{1}\right), \ldots, f\left(b_{n}\right)\right) & & \left(f^{\circ} \text { is a homomorphism extending } f\right) \\
& \in t^{\mathcal{P}(W)}\left(\bar{f}\left(b_{1}\right), \ldots, \bar{f}\left(b_{n}\right)\right) & & (\circ \text { in } \mathcal{P}(W) \text { is element-wise }) \\
& \subseteq t^{\mathbf{W}^{+}}\left(\bar{f}\left(b_{1}\right), \ldots, \bar{f}\left(b_{n}\right)\right)=\bar{f}^{\circ}(x) & & \left(\gamma_{N} \text { is a closure operator }\right) .
\end{aligned}
$$

So $f^{\circ}(x) \in \bar{f}^{\circ}(x) \subseteq \bar{f}(a) \subseteq\{f(a)\}^{\triangleleft}$; hence $f^{\circ}(x) \in\{f(a)\}^{\triangleleft}$, i.e. $f^{\circ}(x) N f(a)$.

Corollary 3.3 (Adequacy). If a sequent is valid in RLUG, then it is valid in all cut-free Gentzen ru-frames $(\mathbf{W}, \mathbf{B})$, where $\mathbf{B}$ is a total algebra.

Together with Lemmas 2.2, 2.3 we obtain the following well-known result that can also be proved in a routine way without the use of Gentzen frames.

Corollary 3.4 (Completeness). A sequent is provable in GL iff it is valid in RLUG. The same holds for the systems $\mathbf{G L}_{\mathbf{a}}$ and $\mathbf{F L}$ with respect to $\mathrm{FL}$.

Corollary 3.5. The free algebra in RLUG is embeddable in $\mathbf{W}_{\mathbf{G L}}^{+}$.

Proof. By Corollary 2.7, the map $a \mapsto\{a\}^{\triangleleft}$ from $\mathbf{F m}$ to $\mathbf{W}_{\mathbf{G} \mathbf{L}}^{+}$is a homomorphism. The pair $(a, b)$ is in its kernel iff $\{a\}^{\triangleleft}=\{b\}^{\triangleleft}$, iff $a \Rightarrow b$ and $b \Rightarrow a$ are provable in GL, namely iff $a=b$ holds in RLUG, by Corollary 3.4. Thus, by the first Isomorphism Theorem, the free (Tarski-Lindenbaum) algebra in RLUG is isomorphic to the image of the above homomorphism.

A Gentzen system (such as $\mathbf{G L}$ or $\mathbf{F L}$ ) is said to have the cut-elimination property if it proves the same sequents as its cut-free version. Note that only the admissibility of the rules (of GL) is used in the proof below. (An inference rule is admissible if its addition does not lead to more provable sequents.) Standard proofs of this well-known result are usually longer and syntax based rather than with the present semantic approach.

Corollary 3.6 (Cut elimination). Cut elimination holds for $\mathbf{G L}, \mathbf{G L}_{\mathbf{a}}$ and $\mathbf{F L}$. 
Proof. For GL, note that if $\vdash_{\mathbf{G L}} x \Rightarrow a$, then $\models_{\mathrm{RLUG}} x \Rightarrow a$, so $\mathbf{W}_{\mathrm{cfG}}^{+} \models x \Rightarrow a$, where $\mathbf{W}_{\text {cfGL }}$ is the frame associated with cut-free $\mathbf{G L}$. By Theorem 3.2, we have $\left(\mathbf{W}_{\mathrm{cfGL}}, \mathbf{F m}\right) \models x \Rightarrow a$, so $\vdash_{\mathrm{cfGL}} x \Rightarrow a$.

Decidability. The cut-elimination property allows for an effective decision procedure for determining if a given sequent is provable or not. The algorithm is based on a standard exhaustive proof search in the cut-free system by constructing all possible proof figures with the sequent as the end result. This process terminates since the number of connectives decreases as we read the proof figure upward.

Corollary 3.7. Each of GL, GL $\mathbf{a}$, FL has a decidable set of provable sequents. The varieties RLUG and FL have decidable equational theories.

Cut elimination with simple structural rules. In [14, and independently in [23. cut elimination is shown for extensions of GL by certain structural rules, including the basic ones. In Corollary 3.13 we extend these results even further.

Let $t_{0}, t_{1}, \ldots, t_{n}$ be elements of the absolutely free algebra in the signature $\{\circ, \varepsilon\}$ over a countable set of variables, $t_{0}$ a linear term, and $(\mathbf{W}, \mathbf{B})$ a Gentzen frame. Also $t_{i}^{\mathbf{W}}$ denotes the term function on $\mathbf{W}$ defined by $t_{i}$. A simple rule is of the form

$$
\frac{t_{1} N q \quad \cdots \quad t_{n} N q}{t_{0} N q}[\mathrm{r}]
$$

where $q$ is a variable not occurring in $t_{0}, t_{1}, \ldots, t_{n} .(\mathbf{W}, \mathbf{B})$ satisfies $[\mathrm{r}]$ if for all $z \in W^{\prime}$, and for all sequences $\bar{x}$ of elements of $W$ matching the variables involved in $t_{0}, t_{1}, \ldots, t_{n}$, the conjunction of the conditions $t_{i}^{\mathbf{W}}(\bar{x}) N z$, for $i \in\{1, \ldots, n\}$, implies $t_{0}^{\mathbf{W}}(\bar{x}) N z$. The definition of a simple rule for $\left(\mathbf{W}_{\mathbf{G L}}, \mathbf{F m}\right)$ motivates the following definition of a simple rule in $\mathbf{G L}$ (subject to the same restrictions as above):

$$
\frac{u\left(t_{1}\right) \Rightarrow a \quad \cdots \quad u\left(t_{n}\right) \Rightarrow a}{u\left(t_{0}\right) \Rightarrow a}(\mathrm{r})
$$

E.g., contraction, integrality and associativity are simple structural rules. Clearly, the frame $\left(\mathbf{W}_{\mathbf{G L}_{(r)}}, \mathbf{F m}\right)$ satisfies the corresponding simple rule $[r]$.

Note that $t_{i}$ and the term function $t_{i}^{\mathbf{F m}}$ on the algebra $(F m, \cdot 1)$ are interdefinable, by fixing a bijection between the variables of the two related algebras. The same holds for $(\mathrm{r})$ and the inequality $e(\mathrm{r})=\left(t_{0}^{\mathbf{F} \mathbf{m}_{\mathcal{L}}} \leq t_{1}^{\mathbf{F m}} \vee \cdots \vee t_{n}^{\mathbf{F m}}\right)$, called simple.

In RLUG, every equation $e$ over $\{\mathrm{V}, \cdot, 1\}$ is equivalent to a conjunction of inequalities of the form above. To show this we distribute all products over all joins to reach a form $s_{1} \vee \cdots \vee s_{m}=t_{1} \vee \cdots \vee t_{n}$, where $s_{i}, t_{j}$ are unital groupoid terms. Such an equation is in turn equivalent to the conjunction of the two inequalities $s_{1} \vee \cdots \vee s_{m} \leq t_{1} \vee \cdots \vee t_{n}$ and $t_{1} \vee \cdots \vee t_{n} \leq s_{1} \vee \cdots \vee s_{m}$. Finally, the first one (and likewise the second) is equivalent to the conjunctions of the inequalities $s_{j} \leq t_{1} \vee \cdots \vee t_{n}$. We now rewrite each of the conjuncts, say $s \leq t_{1} \vee \cdots \vee t_{n}$, in a form for which $s$ is a linear term. For each variable $x$ that appears $k>1$ times in $s$, we replace each occurrence of $x$ in the equation by $x_{1} \vee x_{2} \vee \cdots \vee x_{k}$, where $x_{1}, \ldots, x_{k}$ are fresh variables. As multiplication distributes over join, the new equation can be written in the form $s_{1}^{\prime} \vee \cdots \vee s_{p}^{\prime} \leq t_{1}^{\prime} \vee \cdots \vee t_{q}^{\prime}$, where all the terms are products of variables. Let $s_{l}^{\prime}$ be one of the $k$ !-many linear terms among $s_{1}^{\prime}, \ldots, s_{p}^{\prime}$. The last equation clearly implies the equation $s_{l}^{\prime} \leq t_{1}^{\prime} \vee \cdots \vee t_{q}^{\prime}$, but it is actually 
equivalent to it, as the latter implies $s \leq t_{1} \vee \cdots \vee t_{n}$ by setting all duplicate copies of each variable equal to each other. For an equation $e, \mathrm{R}(e)$ denotes the set of rules associated with each of these conjuncts obtained from $e$, as described above.

In the way of transforming simple rules to equations over $\{\mathrm{V}, \cdot, 1\}$ and vice versa we established the following lemma, whose proof-theoretic analogue appears in [23].

Lemma 3.8. Every equation over $\{\vee, \cdot, 1\}$ is equivalent to a conjunction of simple equations.

Lemma 3.9. Every equation e over $\{\bigvee, \cdot, 1\}$ is equivalent, relative to RLUG, to $\mathrm{R}(e)$. More precisely, for every $\mathbf{G} \in \mathrm{RLUG}, \mathbf{G}$ satisfies e iff $\mathbf{W}_{\mathbf{G}}$ satisfies $\mathrm{R}(e)$.

Proof. It suffices to show the lemma for the case where $e$ is of the form $t_{0}^{\mathbf{F m}} \leq$ $t_{1}^{\mathbf{F m}} \vee \cdots \vee t_{n}^{\mathbf{F m}}$. Clearly $\mathbf{W}_{\mathbf{G}}$ satisfies $\mathrm{R}(e)$ iff $\mathbf{G}$ satisfies the implication: if $t_{i}^{\mathbf{F m}} \leq \bar{z}$ for all $i \in\{1, \ldots, n\}$, then $t_{0}^{\mathbf{F m}} \leq z$, for all propositional variables $z$, which by lattice-theoretic considerations is equivalent to $e$.

Theorem 3.10. Let $(\mathbf{W}, \mathbf{B})$ be a cut-free Gentzen frame and let $e$ be an equation over $\{\vee, \cdot, 1\}$. Then $(\mathbf{W}, \mathbf{B})$ satisfies $\mathrm{R}(e)$ iff $\mathbf{W}^{+}$satisfies e.

Proof. Clearly it suffices to prove the lemma for the case where $e$ is simple, namely of the form $t_{0}^{\mathbf{F m}} \leq t_{1}^{\mathbf{F m}} \vee \cdots \vee t_{n}^{\mathbf{F m}}$, where $t_{0}$ is linear.

Assume that $(\mathbf{W}, \mathbf{B})$ satisfies $\mathrm{R}(e)$. Let $\bar{X}=\left(X_{j}\right)_{j \in J}$ be a sequence of elements in $\mathbf{W}^{+}$. We will show that $e^{\mathbf{W}^{+}}(\bar{X})$ holds, i.e., $t_{0}^{\mathbf{W}^{+}}(\bar{X}) \subseteq t_{1}^{\mathbf{W}^{+}}(\bar{X}) \vee \cdots \vee t_{n}^{\mathbf{W}^{+}}(\bar{X})$. Assume that $t_{1}^{\mathbf{W}^{+}}(\bar{X}) \vee \cdots \vee t_{n}^{\mathbf{W}^{+}}(\bar{X}) \subseteq\{z\}^{\triangleleft}$, for some $z \in W^{\prime}$. It suffices to show that $t_{0}^{\mathbf{W}^{+}}(\bar{X}) \subseteq\{z\} \triangleleft$. We have $t_{1}^{\mathbf{W}^{+}}(\bar{X}) \cup \cdots \cup t_{n}^{\mathbf{W}^{+}}(\bar{X}) \subseteq t_{1}^{\mathbf{W}^{+}}(\bar{X}) \vee \cdots \vee t_{n}^{\mathbf{W}^{+}}(\bar{X})$, so for every $i \in\{1, \ldots, n\}$, we have $t_{i}^{\mathbf{W}^{+}}(\bar{X}) \subseteq\{z\} \triangleleft$. If $x_{j} \in X_{j}$, for all $j \in J$ (we abbreviate this by $\bar{x} \in \bar{X})$ and $\bar{x}=\left(x_{j}\right)_{j \in J}$, then

$$
\begin{aligned}
t_{i}^{\mathbf{W}}(\bar{x})=t_{i}^{\mathbf{W}}\left(\left(x_{j}\right)_{j \in J}\right) & \in t_{i}^{\mathcal{P}(\mathbf{W})}\left(\left(\left\{x_{j}\right\}\right)_{j \in J}\right) & & \text { (by the definition of } \circ \text { in } \mathcal{P}(\mathbf{W})) \\
& \subseteq t_{i}^{\mathcal{P}(\mathbf{W})}(\bar{X}) & & \text { (operations are element-wise) } \\
& \subseteq \gamma_{N}\left(t_{i}^{\mathcal{P}(\mathbf{W})}(\bar{X})\right) & & \left(\gamma_{N}\right. \text { is a closure operator) } \\
& =t_{i}^{\mathbf{W}^{+}}(\bar{X}) \subseteq\{z\}^{\triangleleft} & & \text { (Lemma 1.4(ii)). }
\end{aligned}
$$

It follows that $t_{i}^{\mathbf{W}}(\bar{x}) N z$, for all $i \in\{1, \ldots, n\}$. Hence $t_{0}^{\mathbf{W}}(\bar{x}) N z$, by $\mathrm{R}(e)$, and $t_{0}^{\mathbf{W}}(\bar{x}) \in\{z\}^{\triangleleft}$, for all $\bar{x} \in \bar{X}$. Since $t_{0}$ is a linear term, we obtain $t_{0}^{\mathcal{P}(\mathbf{W})}(\bar{X}) \subseteq\{z\}^{\triangleleft}$. Since $\{z\}^{\triangleleft}$ is a closed set, we have $t_{0}^{\mathbf{W}^{+}}(\bar{X})=\gamma_{N}\left(t_{0}^{\mathcal{P}(\mathbf{W})}(\bar{X})\right) \subseteq\{z\}^{\triangleleft}$.

Conversely, assume that $\mathbf{W}^{+}$satisfies $e$. For every sequence $\bar{X}=\left(X_{j}\right)_{j \in J}$ of elements in $\mathbf{W}^{+}$, we have $t_{0}^{\mathbf{W}^{+}}(\bar{X}) \subseteq t_{1}^{\mathbf{W}^{+}}(\bar{X}) \vee \cdots \vee t_{n}^{\mathbf{W}^{+}}(\bar{X})$. In particular, for $X_{j}=\gamma_{N}\left(\left\{x_{j}\right\}\right)$, where $x_{j} \in W$, we have

$$
t_{0}^{\mathbf{W}^{+}}\left(\left(\gamma_{N}\left(\left\{x_{j}\right\}\right)\right)_{j \in J}\right) \subseteq t_{1}^{\mathbf{W}^{+}}\left(\left(\gamma_{N}\left(\left\{x_{j}\right\}\right)\right)_{j \in J}\right) \vee \cdots \vee t_{n}^{\mathbf{W}^{+}}\left(\left(\gamma_{N}\left(\left\{x_{j}\right\}\right)\right)_{j \in J}\right)
$$

By Lemma 1.4(ii), we obtain successively

$$
\begin{gathered}
\gamma_{N}\left(t_{0}^{\mathcal{P}(\mathbf{W})}\left(\left(\left\{x_{j}\right\}\right)_{j \in J}\right)\right) \subseteq \gamma_{N}\left(t_{1}^{\mathcal{P}(\mathbf{W})}\left(\left(\left\{x_{j}\right\}\right)_{j \in J}\right) \cup \cdots \cup t_{n}^{\mathcal{P}(\mathbf{W})}\left(\left(\left\{x_{j}\right\}\right)_{j \in J}\right)\right) \\
\gamma_{N}\left(\left\{t_{0}^{\mathbf{W}}(\bar{x})\right\}\right) \subseteq \gamma_{N}\left(\left\{t_{1}^{\mathbf{W}}(\bar{x}), \ldots, t_{n}^{\mathbf{W}}(\bar{x})\right\}\right)
\end{gathered}
$$


Therefore, for all $z \in W^{\prime}, \gamma_{N}\left(\left\{t_{1}^{\mathbf{W}}(\bar{x}), \ldots, t_{n}^{\mathbf{W}}(\bar{x})\right\}\right) \subseteq\{z\} \triangleleft$ implies $\gamma_{N}\left(\left\{t_{0}^{\mathbf{W}}(\bar{x})\right\}\right) \subseteq$ $\{z\}^{\triangleleft}$, namely $\left\{t_{1}^{\mathbf{W}}(\bar{x}), \ldots, t_{n}^{\mathbf{W}}(\bar{x})\right\} \subseteq\{z\}^{\triangleleft}$ implies $t_{0}^{\mathbf{W}}(\bar{x}) \in\{z\}^{\triangleleft}$. Consequently, $\left(t_{1}^{\mathbf{W}}(\bar{x}) N z\right.$ and $\left.\ldots t_{n}^{\mathbf{W}}(\bar{x}) N z\right)$ implies $t_{0}^{\mathbf{W}}(\bar{x}) N z$, and $\mathrm{R}(e)$ holds in $(\mathbf{W}, \mathbf{B})$.

It follows from Lemma 3.9 that if $(\mathbf{W}, \mathbf{B})$ is a Gentzen frame, then $\mathbf{W}^{+}$satisfies $e$ iff $\mathbf{W}^{+}$satisfies $\mathrm{R}(e)$. We say that a set $\mathrm{R}$ of rules is preserved by $\left({ }_{-}\right)^{+}$, if for every cut-free Gentzen frame $(\mathbf{W}, \mathbf{B})$, if $(\mathbf{W}, \mathbf{B})$ satisfies $R$, then $\mathbf{W}^{+}$satisfies $\mathrm{R}$.

Corollary 3.11. All simple rules are preserved by $\left({ }_{-}\right)^{+}$.

The rules of exchange, weakening, contraction and associativity are preserved by $\left({ }_{-}\right)^{+}$. For a set $\mathrm{R}$ of rules an $\mathrm{R}-$ Gentzen frame is a Gentzen frame that satisfies R. RLUG $\mathrm{R}_{\mathrm{R}}$ denotes the subvariety of RLUG axiomatized by $e(\mathrm{R})=\{e(r): r \in \mathrm{R}\}$. Theorem 3.2 yields:

Corollary 3.12. If a sequent is valid in $\mathrm{RLUG}_{\mathrm{R}}$ for a set of simple rules $\mathrm{R}$, then it is valid in all cut-free $\mathrm{R}-$ Gentzen frames.

Corollary 3.13. The systems $\mathbf{G L}_{\mathrm{R}}, \mathbf{F} \mathbf{L}_{\mathrm{R}}$ enjoy the cut-elimination property, for every set $\mathrm{R}$ of simple rules, and in particular for the set $\mathrm{R}=\mathrm{R}(e)$ with simple rules for an equation e over $\{\vee, \cdot, 1\}$, e.g., for $\mathrm{R} \subseteq\{a, e, c, i\}$.

Proof. Every sequent valid in the frame $\mathbf{W}_{\mathbf{G L}_{\mathrm{R}}}$ is also valid in $\mathrm{RLUG}$. By Corollary 3.12 it is valid in the frame $\mathbf{W}_{\mathrm{cfG}_{\mathbf{R}}}$ associated with the cut-free system.

Corollary 3.14. The free algebra in $\mathrm{RLUG}$ is embeddable in $\mathbf{W}_{\mathbf{G}_{\mathbf{R}}}^{+}$.

Finite model property. We say that a Gentzen system has the finite model property (FMP), if for every sequent that is not provable there exists a finite countermodel. We will show the FMP for extensions by simple rules. Recall that a variety (equivalently, an equational class) is a class of algebras closed under homomorphic images, subalgebras and direct products. It has the FMP if every nonvalid equation is actually falsified in a finite algebra in the variety. Clearly a variety has the FMP if it is generated by (is the smallest variety that contains) its finite members.

Let $\mathbf{L}$ be a sequent system. For a sequent $s, s^{\leftarrow}$ is the least set of sequents such that $s \in s^{\leftarrow}$ and if $\left(\left\{t_{1}, \ldots, t_{n}\right\}, t\right)$ is an instance of a rule of $\mathbf{L}$ and $t \in s^{\leftarrow}$, then $t_{1}, \ldots, t_{n} \in s^{\leftarrow}$. Clearly $s^{\leftarrow}$ is the set of all sequents involved in an exhaustive proof search for $s$.

We say that a rule in a sequent system does not increase complexity if for each instance of the rule, the complexity of each sequent in the numerator is at most as big as the complexity of the denominator. For simple structural rules, complexity of a sequent can be defined to be, for example, its length (i.e. counting each symbol). As there are only finitely many sequents of the same length, such a rule contributes only a finite number of sequents to $s^{\leftarrow}$. Contraction (c) and (Cut), however, are examples of structural rules that do increase complexity.

A logical rule is a sequent rule that introduces a logical connective (such as $\wedge, \vee, \cdot$, $\backslash, /$ ) on the left or right of the denominator. It is said to have the subformula property if for all instances of the rule, all formulas appearing in the numerator are subformulas of the denominator. If a system has logical rules with the subformula property and the structural rules do not increase complexity, then for any sequent $s$ the set $s^{\leftarrow}$ is finite.

Theorem 3.15. The systems GL, FL as well as their extensions with simple rules that do not increase complexity have the FMP. 
Proof. For GL, we consider the frame $\mathbf{W}_{\mathbf{G L}}=\left(W, W^{\prime}, N, \circ, \|, / / E\right)$ and a sequent $s$ that is not provable in GL. Let $N^{\prime}$ be the relation defined by

$$
x N^{\prime}(u, a) \quad \text { iff } \quad x N(u, a) \text { or } \quad(u(x) \Rightarrow a) \notin s^{\leftarrow} .
$$

To see that $N^{\prime}$ is nuclear, let $x, y \in W,(u, a) \in W^{\prime}$ and define $v(z)=u(z \circ y)$, for all $z \in W$. We have $x \circ y N^{\prime}(u, a)$ iff $x \circ y N(u, a)$ or $[u(x \circ y) \Rightarrow a] \notin s^{\leftarrow}$ iff $x N(v, a)$ or $(v(x) \Rightarrow a) \notin s^{\leftarrow}$ iff $x N^{\prime}(v, a)$. Also, $N^{\prime}$ satisfies the conditions GN. Indeed, let $\left(\left\{t_{1}, t_{2}\right\}, t_{0}\right)$ be a rule of $\mathbf{G N}$ and assume that $t_{1}, t_{2} \in N^{\prime}$. If $t_{1}, t_{2} \in N$, then $t_{0} \in N$ (since $N$ satisfies GN) and $t_{0} \in N^{\prime}$. Otherwise, $t_{1}^{\prime} \notin s^{\leftarrow}$ or $t_{2}^{\prime} \notin s^{\leftarrow}$. Here, if $t_{i}=(x,(u, a))$, by $t_{i}^{\prime}$ we denote the sequent $u(x) \Rightarrow a$. By the (contrapositive of the) second condition for $s^{\leftarrow}$, we have $t_{0}^{\prime} \notin s^{\leftarrow}$ and thus again $t_{0} \in N^{\prime}$. So, $\left(\mathbf{W}_{s}, \mathbf{F m}\right)$ is a Gentzen frame, where $\mathbf{W}_{s}=\left(W, W^{\prime}, N^{\prime}, \circ, \|, / /, E\right)$.

As $s^{\leftarrow}$ is finite, there are finitely many $x, u, a$ such that $(u(x) \Rightarrow a) \in s^{\leftarrow}$. So, the complement $\left(N^{\prime}\right)^{c}$ of $N^{\prime}$ is finite; hence also its image $\operatorname{Im}\left(\left(N^{\prime}\right)^{c}\right)=\left\{z \in W^{\prime}\right.$ : $x\left(N^{\prime}\right)^{c} z$, for some $\left.x \in W\right\}$. If $z \notin \operatorname{Im}\left(\left(N^{\prime}\right)^{c}\right)$, then $W N^{\prime} z$ and $\{z\}^{\triangleleft}=W$, where $\triangleleft$ is with respect to $N^{\prime}$. Therefore, $\{z\}^{\triangleleft} \neq W$ only for the finitely many $z \in \operatorname{Im}\left(\left(N^{\prime}\right)^{c}\right)$. Consequently, there are only finitely many basic closed sets and $\mathbf{W}_{s}^{+}$is finite.

Furthermore, $s$ fails in $\mathbf{W}_{s}^{+}$. Indeed, let $s$ be the sequent $x \Rightarrow a$ and let $b=x^{\mathbf{F m}}$ (i.e. $b$ is the term $x$ with every $\circ$ replaced by $\cdot$ ). Note $x \not X^{\prime} a$, since $x \not D a$ and $(x \Rightarrow a)=s \in s^{\leftarrow}$. Hence $x \notin\{a\}^{\triangleleft}$. However $x \Rightarrow b$ is provable in GL by (.R), so $x \in\{b\}^{\triangleleft}$, and therefore $\{b\}^{\triangleleft} \nsubseteq\{a\}^{\triangleleft}$. Since $\left(\mathbf{W}_{s}, \mathbf{F m}\right)$ is a Gentzen frame, the map $\triangleleft: \mathbf{F m} \rightarrow \mathbf{W}_{s}^{+}$is a homomorphism by Corollary 2.7. Consequently, the inequality $b \leq a$ is not valid in $\mathbf{W}_{s}^{+}$, so neither is the sequent $x \Rightarrow a$.

By performing a (necessarily terminating) exhaustive proof search for a sequent $s$, we either obtain a proof of $s$ or, by using the set $s^{\leftarrow}$, obtained on the way, we construct a finite counterexample $\mathbf{W}_{s}^{+}$. The FMP for $\mathbf{F L}$ was proved in [19] in the setting of phase spaces and for GL it was proved in 14 in the setting of Gentzen matrices. Note that the proof does not make use of specific rules of GL, FL except for $(\cdot R)$; hence the result applies to any such sequent system with the property that $s^{\leftarrow}$ is finite for every sequent $s$, and gives rise to a Gentzen frame $\mathbf{W}$ for which $\mathbf{W}_{s}^{+}$ is a model of the sequent system. Further conditions may be added to the definition of $s^{\leftarrow}$ to enlarge the set in a way that $\mathbf{W}^{+}$becomes smaller. Such a condition is included in [19, and this issue is discussed further in [14.

Finite embeddability property. Let $\mathbf{A}$ be an $r$ lu-groupoid and $\mathbf{B}$ a partial subalgebra of $\mathbf{A}$. Recall that $\left(\mathbf{W}_{\mathbf{A}, \mathbf{B}}, \mathbf{B}\right)$ is a Gentzen frame. By Corollary 2.8 we obtain the following result, which was originally proved in [4].

Corollary 3.16. The map $\left\{{ }_{-}\right\}^{\triangleleft}: \mathbf{B} \rightarrow \mathbf{W}_{\mathbf{A}, \mathbf{B}}^{+}$is an embedding of the partial subalgebra $\mathbf{B}$ of the rlu-groupoid $\mathbf{A}$ into the rlu-groupoid $\mathbf{W}_{\mathbf{A}, \mathbf{B}}^{+}$.

Theorem 3.17. If an equation over $\{\vee, \cdot, 1\}$ is valid in the rlu-groupoid $\mathbf{A}$, then it is also valid in $\mathbf{W}_{\mathbf{A}, \mathbf{B}}^{+}$, for every partial subalgebra $\mathbf{B}$ of $\mathbf{A}$.

Proof. By Lemma 3.8 it is enough to consider simple equations $e$, i.e., of the form $t_{0} \leq t_{1} \vee \cdots \vee t_{n}$, where $t_{0}$ is a linear term. Assume that $e$ is valid in $\mathbf{A}$, and let $\mathbf{B}$ be a partial subalgebra of $\mathbf{A}$. By Theorem 3.10, to show that $e$ is valid in $\mathbf{W}_{\mathbf{A}, \mathbf{B}}^{+}$it is enough to show that the rule $\frac{t_{1} N(u, c) \ldots t_{n} N(u, c)}{t_{0} N(u, c)} \mathrm{R}(e)$ is valid in the Gentzen 
frame $(\mathbf{W}, \mathbf{B})$, namely that if $u\left(t_{i}\right) \leq_{\mathbf{A}} c$, for all $i \in\{1, \ldots, n\}$, then $u\left(t_{0}\right) \leq_{\mathbf{A}} c$; here we abused notation slightly by using, for example, $c$ initially as a metavariable and then as an element of $B$. The latter implication follows directly from the fact that $\mathbf{A}$ satisfies $e$.

A class of algebras $\mathcal{K}$ is said to have the finite embeddability property (FEP) if for every algebra $\mathbf{A}$ in $\mathcal{K}$ and every finite partial subalgebra $\mathbf{B}$ of $\mathbf{A}$, there exists a finite algebra $\mathbf{D}$ in $\mathcal{K}$ such that $\mathbf{B}$ embeds into $\mathbf{D}$.

Theorem 3.18. Every variety of integral rlu-groupoids axiomatized by equations over the signature $\{\vee, \cdot, 1\}$ has the FEP.

Proof. We follow the ideas in [4 to establish the finiteness of $\mathbf{W}_{\mathbf{A}, \mathbf{B}}^{+}$. Let $k$ be the cardinality of the set $B=\left\{b_{1}, \ldots, b_{k}\right\}$ and $F$ the free groupoid with unit over $k$ generators $x_{1}, \ldots, x_{k}$ (so all nonunit elements of $F$ are just products of generators). For $s, t \in F$, we write $s \leq^{F} t$ iff $t$ is obtained from $s$ by deleting some (possibly none) of the generators. We always have $s \leq^{F} 1$. In [4] it is shown that this relation is a partial order on $F$ such that $F$ has no infinite antichains and no infinite ascending chains (it is dually well-ordered), using Higman's Lemma. Moreover, under the above order and multiplication $F$ can be expanded to an integral $r u$-groupoid $\mathbf{F}$.

Let $h: F \rightarrow W$ be the (surjective) homomorphism that extends the assignment $x_{i} \mapsto b_{i}$. Consider the new frame $\mathbf{W}_{\mathbf{A}, \mathbf{B}}^{\mathbf{F}}=\left(F, W^{\prime}, h \circ N,{ }^{\mathbf{F}}, \|_{h}, / / h,\{1\}\right)$, where $x(h \circ N) z$ iff $h(x) N z$, and $x\left\|_{h} z=h(x)\right\| z$ and $z / /{ }_{h} y=z / / h(y)$. It is easy to see that $h \circ N$ is nuclear, so $\mathbf{W}_{\mathbf{A}, \mathbf{B}}^{\mathbf{F}}$ is a residuated frame.

To prove that $\mathbf{W}_{\mathbf{A}, \mathbf{B}}^{+}$is finite, it suffices to prove that it possesses a finite basis of sets $\{z\}^{\triangleleft_{N}}=\{x \in W: x N z\}$, for $z \in W^{\prime}$. As $h$ is surjective, it suffices to show that there are finitely many sets of the form $\{z\}^{\triangleleft}=\{x \in F: x(h \circ N) z\}$, for $z \in W^{\prime}$.

For $x \in F$, and $(u, b) \in W^{\prime}$, we have $x \in\{(u, b)\}^{\triangleleft}$ iff $u(h(x)) \leq b$ iff $h(v(x)) \leq b$, for some $v \in S_{F}$ such that $h(v)=u$, since $h$ is a surjective homomorphism (here we have extended $h$ to a map from $S_{F}$ to $\left.S_{W}\right)$. Equivalently, $v(x) \in h^{-1}\left(\downarrow_{A} b\right)$, for some $v \in h^{-1}(u)$. Now, $h^{-1}\left(\downarrow_{A} b\right)$ is a downset in $F$ and, because $F$ is dually well-ordered, this downset is equal to $\downarrow M_{b}$, for some finite $M_{b} \subseteq F$. So, the above statement is equivalent to $v(x) \leq m$, or to $x \leq \frac{m}{v}$, for some $v \in h^{-1}(u)$ and some $m \in M_{b}$. Here $\frac{m}{v}$ is defined inductively by $\frac{m}{1}=m, \frac{m}{v \cdot x}=\frac{m / x}{v}$ and $\frac{m}{x \cdot v}=\frac{x \backslash m}{v}$, where the divisions are calculated in F. Consequently, $\{(u, b)\} \triangleleft^{\triangleleft}=\downarrow\left\{\frac{m}{v}: m \in M_{b}, h(v)=u\right\}$.

Note that the set $\left\{\frac{m}{v}: m \in M_{b}, b \in B, h(v)=u, u \in S_{W}\right\}$ is finite, being a subset of the finite set $\uparrow \bigcup_{b \in B} M_{b}$, as $m \leq \frac{m}{v}$ (or $v(m) \leq m$ ), by integrality. Thus, there are only finitely many choices for $\{(u, b)\}^{\triangleleft}$.

In [4] the FEP is established for the whole variety of integral $r \ell u$-groupoids, as well as for the associative and the commutative subvarieties, and was extended in 24] to all subvarieties axiomatized by equations of the form $x^{n} \leq x^{m}$. The result also specializes to (always integral) associative and/or commutative $r \ell u(z)$ groupoids. Moreover, as these classes are finitely axiomatized they have decidable universal theories.

All results proved so far also work for poorer signatures that do not include the connectives $\wedge, \backslash, /$, as the proof of Theorem 2.6 handles each connective separately. However, $\vee, \cdot, 1$ need to be present, to support the linearization process. 
TABLE 3. The system InGL.

$$
\begin{aligned}
& \frac{x \Rightarrow a \quad a \Rightarrow z}{x \Rightarrow z}(\mathrm{CUT}) \quad \overline{a \Rightarrow a}(\mathrm{Id}) \quad \frac{a \circ b \Rightarrow z}{a \cdot b \Rightarrow z}(\cdot \mathrm{L}) \quad \frac{x \Rightarrow a \quad y \Rightarrow b}{x \circ y \Rightarrow a \cdot b}(\cdot \mathrm{R}) \\
& \frac{a \Rightarrow z}{a \wedge b \Rightarrow z}(\wedge \mathrm{L} \ell) \quad \frac{b \Rightarrow z}{a \wedge b \Rightarrow z}(\wedge \mathrm{L} r) \quad \frac{x \Rightarrow a \quad x \Rightarrow b}{x \Rightarrow a \wedge b}(\wedge \mathrm{R}) \\
& \frac{a \Rightarrow z \quad b \Rightarrow z}{a \vee b \Rightarrow z}(\vee \mathrm{L}) \quad \frac{x \Rightarrow a}{x \Rightarrow a \vee b}(\vee \mathrm{R} \ell) \quad \frac{x \Rightarrow b}{x \Rightarrow a \vee b}(\vee \mathrm{R} r) \\
& \frac{\varepsilon \Rightarrow z}{1 \Rightarrow z}(1 \mathrm{~L}) \quad \frac{}{\varepsilon \Rightarrow 1}(1 \mathrm{R}) \quad \frac{x \circ y \Rightarrow z}{\overline{y \Rightarrow x^{\sim} \circ z}}(\sim) \quad \frac{x \circ y \Rightarrow z}{\overline{x \Rightarrow z \circ y^{-}}}\left({ }^{-}\right) \\
& \frac{a^{\sim} \Rightarrow z}{\sim a \Rightarrow z}(\sim \mathrm{L}) \quad \frac{x \Rightarrow a^{\sim}}{x \Rightarrow \sim a}(\sim \mathrm{R}) \quad \frac{a^{-} \Rightarrow z}{-a \Rightarrow z}(-\mathrm{L}) \quad \frac{x \Rightarrow a^{-}}{x \Rightarrow-a}(-\mathrm{R})
\end{aligned}
$$

\section{INVOLUTIVE FRAMES}

Definition and examples. An involutive (residuated) frame is a structure of the form $\mathbf{W}=\left(W, N, \circ, E,^{\sim},^{-}\right)$, where $N \subseteq W^{2}, \circ \subseteq W^{3}, E \subseteq W$ and ${ }^{\sim},-$ are unary operations on $W$ (extended to subsets via $Z^{-}=\left\{z^{-}: z \in Z\right\}$ and $\left.Z^{\sim}=\left\{z^{\sim}: z \in Z\right\}\right)$ such that

(i) $(W, W, N, \circ, \|, / /, E)$ is an $r u$-frame with $x \| y=\left(y^{-} \circ x\right)^{\sim}, y / / x=\left(x \circ y^{\sim}\right)^{-}$,

(ii) $x^{\sim-}=x=x^{-\sim}$ and $\left(y^{\sim} \circ x^{\sim}\right)^{-}=\left(y^{-} \circ x^{-}\right)^{\sim}$ for all $x, y \in W$,

(iii) $(x \circ E)^{\triangleright}=\{x\}^{\triangleright}=(E \circ x)^{\triangleright}$, for all $x \in W$ (weak unit).

The definition of the relations $\|, / /$ is more intuitive if one defines a ternary relation $\oplus$ by $x \oplus y=\left(y^{\sim} \circ x^{\sim}\right)^{-}=\left(y^{-} \circ x^{-}\right)^{\sim}$, since then $x \| y=x^{\sim} \oplus y$ and $y / / x=y \oplus x^{-}$. We also recall two related algebraic notions:

An involutive groupoid with unit is an algebra $\mathbf{G}=\left(G, \circ, 1,,^{-}\right)$such that $(G, \circ, 1)$ is a groupoid with unit (so $\circ$ is an operation rather than a relation) that satisfies $x^{\sim-}=x=x^{-\sim}$ and $(x \circ y)^{\sim}=y^{\sim} \circ x^{\sim}$. Hence $(x \circ y)^{-}=y^{-} \circ x^{-}$, $\left(y^{\sim} \circ x^{\sim}\right)^{-}=\left(y^{-} \circ x^{-}\right)^{\sim}$ and $1^{\sim}=1^{-}=1$.

A weakly involutive groupoid with unit is an algebra $\mathbf{G}=\left(G, \circ, 1, \sim,^{-}\right)$such that $(G, \circ, 1)$ is a groupoid with unit that satisfies $x^{\sim-}=x=x^{-\sim}$ and $\left(y^{\sim} \circ x^{\sim}\right)^{-}=$ $\left(y^{-} \circ x^{-}\right)^{\sim}$. It follows that $(x \circ y)^{\sim \sim}=x^{\sim \sim} \circ y^{\sim \sim},(x \circ y)^{--}=x^{--} \circ y^{--}$and $1^{\sim}=1^{-}$. If an operation $\oplus$ is defined by $x \oplus y=\left(y^{\sim} \circ x^{\sim}\right)^{-}$, then $\left(G, \oplus, 1^{\sim},,^{\sim}\right)$ is also a weakly involutive groupoid with unit.

The Gentzen system InGL is defined in Table 3 and is an involutive analogue of GL. Formulas of InGL are defined with respect to the language $\{\wedge, \vee, \cdot \sim,-, 1\}$. Whereas GL has connectives $\backslash, /$, they are definable in InGL by $x \backslash y=\sim(-y \cdot x)$, $y / x=-(x \cdot \sim y)$. The dual of $\cdot$ is defined by the term $x+y=\sim(-y \cdot-x)$, and $0=\sim 1$. Also note that there are four structural (or external) connectives ("commas"): $\circ$, $\varepsilon, \sim,-$ (the latter two are written postfix). Another important difference is that the rules are written without any explicit reference to a context $u$. However, the bidirectional structural rules $(\sim)$ and $\left(^{-}\right)$allow any context to be moved back and forth between the two sides of a sequent.

Metavariables $a, b, c$ range over formulas and $x, y$ over elements of the free involutive groupoid with unit over the set of formulas. We also consider the Gentzen system InFL, defined by taking the free involutive monoid, instead. In both cases 
we can assume that terms are normalized so that the operations $\circ, \varepsilon$ do not appear in the scope of $\sim,-$. E.g. we always have $\varepsilon^{\sim}=\varepsilon^{-}=\varepsilon$, and in InFL, for $x=a_{1} \circ \cdots \circ a_{n}$ we have $x^{\sim}=a_{n}^{\sim} \circ \cdots \circ a_{1}^{\sim}$ and $x^{-}=a_{n}^{-} \circ \cdots \circ a_{1}^{-}$. Note that as $\mathbf{F L}$ is equivalent to $\mathbf{G L}_{\mathbf{a}}^{0}$, InFL is equivalent to $\mathbf{I n} \mathbf{G L}_{\mathbf{a}}^{0}$. For normalized $x, y$, a sequent $x \Rightarrow y$ is valid in an involutive $r \ell u$-groupoid if for any assignment $f$ to the variables, we have $f(x) \leq f(y)$, where $\circ, \varepsilon, \sim$, - are interpreted as $\cdot, 1, \sim,-$ in $x$ and as $+, 0, \sim,-$ in $y$. Further discussion of validity appears in the subsection on cut elimination below. The next result is proved by checking that each InGL-rule (resp. InFL-rule) is valid in involutive $r \ell u$-groupoids (resp. InFL-algebras).

Lemma 4.1 (Soundness). Every sequent that is provable in InGL is valid in all

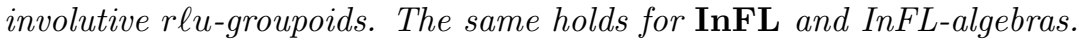

The following rules are derivable in InGL and hence can be added conservatively:

$$
\frac{a \Rightarrow z \quad b \Rightarrow w}{a+b \Rightarrow z \circ w}(+\mathrm{L}) \quad \frac{x \Rightarrow a \circ b}{x \Rightarrow a+b}(+\mathrm{R}) \quad \overline{0 \Rightarrow \varepsilon}(0 \mathrm{~L}) \quad \frac{x \Rightarrow \varepsilon}{x \Rightarrow 0}(0 \mathrm{R}) .
$$

The involutive frames $\mathbf{W}_{\text {InGL }}$ and $\mathbf{W}_{\text {InFL }}$ are defined in the obvious way, where $x N z$ iff $\vdash x \Rightarrow z$. In both cases $\left(W, \circ, \varepsilon, \sim^{\sim},-\right)$ is an involutive groupoid with unit and $x \circ y=x \oplus y$. It is easy to see that if $\mathbf{G}$ is an involutive $r \ell u$-groupoid, then $\mathbf{W}_{\mathbf{G}}=(G, \leq, \cdot\{1\}, \sim,-)$ is an involutive frame. Note that $(G, \cdot, 1, \sim,-)$ is not an involutive groupoid with unit, since in general $x \cdot y \neq-(\sim x \cdot \sim y)$.

Galois algebra. For $X \subseteq W$, we define $-X=X^{-\triangleleft}$ and $\sim X=X^{\sim \triangleleft}$. Note that $-X$ and $\sim X$ are Galois closed sets.

Lemma 4.2. If $\mathbf{W}$ is an involutive frame, $x, y \in W$ and $X, Y, Z \subseteq W$, then:

(i) $x N y^{-}$iff $y N x^{\sim}$.

(ii) $\sim E=-E$.

(iii) $-Y=Y^{\triangleright-}=Y^{-\triangleleft}$ and $\sim Y=Y^{\triangleright \sim}=Y^{\sim \triangleleft}$.

(iv) $X \circ Y \subseteq Z$ iff $Y \subseteq \sim(-Z \circ X)$ iff $X \subseteq-(Y \circ \sim Z)$, if $Z$ is Galois closed.

Proof. (i) $x N y^{-}$iff $x \circ E N y^{-}$iff $E N x^{\sim} \oplus y^{-}$iff $E \circ y N x^{\sim}$ iff $y N x^{\sim}$. (ii) $x \in \sim E=E^{\sim \triangleleft}$ iff $x N E^{\sim}$ iff $x \circ E N E^{\sim}$ iff $x N E^{\sim} \oplus E^{-}$iff $E \circ x N E^{-}$iff $x N E^{-}$iff $x \in E^{-\triangleleft}=-E$. (iii) For all $x \in W$, we have $x \in Y^{-\triangleleft}$ iff $x N Y^{-}$ iff $Y N x^{\sim}$ iff $x^{\sim} \in Y^{\triangleright}$ iff $x \in Y^{\triangleright-}$, by (i). (iv) We have $X \circ Y \subseteq Z=Z^{\triangleright \triangleleft}$ iff $X \circ Y N Z^{\triangleright}$ iff $Y N X^{\sim} \oplus Z^{\triangleright}$ iff $Y \subseteq\left(X^{\sim} \oplus Z^{\triangleright}\right)^{\triangleleft}$. Also, $\left(X^{\sim} \oplus Z^{\triangleright}\right)^{\triangleleft}=$ $\left(X^{\sim} \oplus Z^{\triangleright}\right)^{-\sim \triangleleft}=\left(Z^{\triangleright-} \circ X^{\sim-}\right)^{\sim \triangleleft}=\sim(-Z \circ X)$, by (iii).

For an involutive frame $\mathbf{W}$, we know that $(W, W, N, \circ,\|,\|, E)^{+}$is an $r \ell$-groupoid, with unit $1=E$. By Lemma 4.2 (ii), $\sim 1=-1$, and this common value is denoted by 0 . We write $\mathbf{W}^{+}$for the expansion of that $r \ell u$-groupoid with the element 0 .

Corollary 4.3. For any involutive frame $\mathbf{W}, \mathbf{W}^{+}$is an involutive rlu-groupoid.

Proof. We will show that the operations $\sim$ and - , defined independently of the operations in an $r \ell u$-groupoid, coincide with the usual negations of an $r \ell u$-groupoid, namely that $\sim Z=Z \backslash 0$ and $-Z=0 / Z$, for all $Z \in \mathbf{W}^{+}$. We have $\sim-Z=$ $Z^{\triangleright-\sim \triangleleft}=Z^{\triangleright \triangleleft}=Z$, by Lemma 4.2(iii). Likewise, $-\sim Z=Z$. Finally, by Lemma 4.2(iv), $Z \backslash 0=\sim(-0 \circ Z)=(E \circ Z)^{\triangleright \sim}=Z^{\triangleright \sim}=\sim Z$, and likewise $0 / Z=-Z$. Consequently, $\mathbf{W}^{+}$is an involutive $r \ell u$-groupoid. 
Involutive Gentzen frames. An involutive Gentzen frame is a pair (W, B), where

(i) $\mathbf{W}=\left(W, N, \circ,\{\varepsilon\}, \sim{ }^{-}\right)$is an involutive frame, where $\circ$ is an operation,

(ii) $\mathbf{B}$ is a partial algebra of the type of $\ln G L$,

(iii) $B$ is a subset of $W$ that generates $(W, \circ, \varepsilon, \sim,-)$ and

(iv) $N$ satisfies GN (omitting the $\backslash, /$-rules) and the four rules below, for all $a, b \in B, x, y \in W$ and $z \in W^{\prime}:$

$\frac{a^{\sim} N z}{\sim a N z}[\sim \mathrm{L}] \quad \frac{x N a^{\sim}}{x N \sim a}[\sim \mathrm{R}] \quad \frac{a^{-} N z}{-a N z}[-\mathrm{L}] \quad \frac{x N a^{-}}{x N-a}[-\mathrm{R}]$.

For example, $\left(\mathbf{W}_{\mathbf{I n G L}}, \mathbf{F m}\right)$ and $\left(\mathbf{W}_{\mathbf{I n F L}}, \mathbf{F m}\right)$ are involutive Gentzen frames.

Theorem 4.4. Let $(\mathbf{W}, \mathbf{B})$ be a cut-free involutive Gentzen frame. Then the conditions in Theorem [2.6] hold. Moreover, for all $a \in B, X \in \mathbf{W}^{+}$, if $\sim a$ and $-a$ are defined and $a \in X \subseteq\{a\}^{\triangleleft}$, then

(i) $\sim a \in \sim X \subseteq\{\sim a\}^{\triangleleft}$ and $-a \in-X \subseteq\{-a\}^{\triangleleft}$.

(ii) In particular, $\sim a \in \sim\{a\}^{\triangleleft} \subseteq\{\sim a\}^{\triangleleft}$ and $-a \in-\{a\}^{\triangleleft} \subseteq\{-a\}^{\triangleleft}$.

(iii) If $N$ also satisfies [CUT], then $\sim\{a\}^{\triangleleft}=\{\sim a\}^{\triangleleft}$ and $-\{a\}^{\triangleleft}=\{-a\}^{\triangleleft}$.

Proof. If $\mathbf{B}_{r}$ is the 0 -free reduct of $\mathbf{B}$ and $\mathbf{W}_{r}=(W, W, N, \circ, \mathbb{N}, \|,\{\varepsilon\})$, then $\left(\mathbf{W}_{r}, \mathbf{B}_{r}\right)$ satisfies the conditions of a Gentzen frame except for the fact that $B_{r}$ generates $(W, \circ, \varepsilon)$. Nevertheless, this condition is not used in the proof of Theorem 2.6. Since $\mathbf{W}^{+}$is simply the expansion of $\mathbf{W}_{r}^{+}$by 0 , the conclusion of Theorem 2.6 holds.

We want to show that $\sim X \subseteq\{\sim a\}^{\triangleleft}$. If $x \in \sim X=X^{\sim \triangleleft}$, then $x N X^{\sim}$. Since $a \in X$, we have $x N a^{\sim}$. By $[\sim \mathrm{R}]$ we obtain $x N \sim a$, or $x \in\{\sim a\}^{\triangleleft}$. To show that $\sim a \in \sim X=X^{\sim \triangleleft}$ we need to prove that $\sim a N X^{\sim}$. We have $X \subseteq\{a\}^{\triangleleft}$, so $X N a$ and $a^{\sim} N X^{\sim}$, by Lemma 4.2(i). Finally, by [ L] we get $\sim a N X^{\sim}$.

Corollary 4.5. If $(\mathbf{W}, \mathbf{B})$ is an involutive Gentzen frame, the map $\left\{{ }_{-}\right\} \triangleleft: \mathbf{B} \rightarrow \mathbf{W}^{+}$ is a homomorphism of the partial algebra $\mathbf{B}$ into the involutive rlu-groupoid $\mathbf{W}^{+}$.

Embedding into the DM-completion and representation.

Corollary 4.6. Let $\mathbf{G}$ be an involutive rlu-groupoid. The $\operatorname{map}\left\{\left\{_{-}\right\}^{\triangleleft}: \mathbf{G} \rightarrow \mathbf{W}_{\mathbf{G}}^{+}\right.$ is an embedding.

Therefore, every involutive FL-algebra can be embedded into a complete one.

Corollary 4.7. Every InFL-algebra is a subalgebra of the nucleus image of the power set of a weakly involutive monoid.

Cut elimination. For this section a (classical) sequent is an element of $\mathrm{Fm}^{i} \times \mathrm{Fm}^{i}$, where $F m^{i}$ denotes the free groupoid with unit over the set of negated formulas. Negated formulas are elements of the form $a^{\sim n}$ or $a^{-n}$, for $a \in F m$, defined by $a^{\sim 0}=a$ and $a^{\sim(n+1)}=\left(a^{\sim n}\right)^{\sim}$ (likewise for $\left.a^{-n}\right)$. Sequents are denoted $x \Rightarrow y$.

Let $(\mathbf{W}, \mathbf{B})$ be an involutive Gentzen frame. Note that every map $f: F m \rightarrow B$ extends to a groupoid (with unit) homomorphism $f^{\circ}:\left(F m^{i}, \circ, \varepsilon\right) \rightarrow(W, \circ, \varepsilon)$ by $f^{\circ}\left(x^{\sim}\right)=\left(f^{\circ}(x)\right)^{\sim}, f^{\circ}\left(x^{-}\right)=\left(f^{\circ}(x)\right)^{-}$and $f^{\circ}\left(x \circ^{F m^{i}} y\right)=f^{\circ}(x) \circ^{W} f^{\circ}(y)$. Likewise, every assignment $f: F m \rightarrow B$ extends to a groupoid (with unit) homomorphism $f^{\oplus}:\left(F m^{i}, \circ, \varepsilon\right) \rightarrow\left(W, \oplus, \varepsilon^{\sim}\right)$ by $f^{\oplus}\left(x^{\sim}\right)=\left(f^{\oplus}(x)\right)^{\sim}, f^{\oplus}\left(x^{-}\right)=\left(f^{\oplus}(x)\right)^{-}$ and $f^{\oplus}\left(x \circ^{F m^{i}} y\right)=f^{\oplus}(x) \oplus^{W} f^{\oplus}(y)$. Also, every homomorphism $f: \mathbf{F m} \rightarrow \mathbf{G}$ into an $\mathcal{L}$-algebra $\mathbf{G}$ extends to a groupoid (with unit) homomorphism $f: \mathbf{F m}^{i} \rightarrow \mathbf{G}$. 
For a total algebra $\mathbf{B}$, a sequent $x \Rightarrow y$ is said to be valid in $(\mathbf{W}, \mathbf{B})$, if for every homomorphism $f: \mathbf{F m} \rightarrow \mathbf{B}$, we have $f^{\circ}(x) N f^{\oplus}(y)$. Note that this agrees with the earlier definition of validity in an involutive $r \ell u$-groupoid.

To illustrate the subtlety of validity we observe that for variables $p, q$ the sequent $p \circ q \Rightarrow p \circ q$ is not provable in InGL, which agrees with the fact that the equation $p \cdot q \leq p+q$ is not valid in $\operatorname{lnGL}$. This is why $\circ$ needs to be interpreted as - in the left-hand side and as + in the right-hand side of a sequent. Also, note that $(p \circ q)^{\sim} \Rightarrow \sim(p+q)$ is provable in InGL, but the equation $\sim(p \cdot q) \leq \sim(p+q)$ is not valid in InGL. On the other hand, $\sim(p+q) \leq \sim(p+q)$ holds, so the above comment about the interpretation of $\circ$ on the two sides of a sequent holds only for occurrences of $\circ$ not under negations. This is the reason why we defined (classical) sequents in such a way that all occurrences of $\circ$ are outermost and all negations are applied to formulas. Note that we have different interpretations of $\circ^{F m^{i}}$ on the two sides of a sequent: it is interpreted by $\circ^{W}$ on the left and by $\oplus^{W}$ on the right. This means that for interpretations in an algebra, ${ }^{\circ m^{i}}$ is interpreted as . and + , respectively. This agrees with the usual practice in semantics for sequent calculus systems. For classical logic we obtain interpretations by using $\wedge$ and $\vee$, respectively.

Also note that the set $W$ in InGL is taken to be the free involutive groupoid with unit. On the other hand, in the definition of an involutive frame the set $W$ is only weakly involutive, whenever the ternary relation $\circ$ is a function. This should not create the impression that we can assume the stronger involutive condition in the definition of a frame. To clarify things, we mention that we could have simply taken the free weakly involutive groupoid with unit $\mathbf{W}=\left(W, \circ,\{\varepsilon\},,^{-}\right)$ on a countable set $X$ of variables. By the weakly involutive law we can define an operation $\oplus$, which can be easily shown to be associative and have as unit the element $\delta=\varepsilon^{\sim}=\varepsilon^{-}$. We can also define iterated negations of elements of $X$, by taking repeated applications of the operations ${ }^{\sim},{ }^{-}$on elements of $X$. The resulting set is denoted $X\urcorner$. It is easy to see that the underlying set of $W$ can be concretely realized as the underlying set $V$ of the free bi-groupoid with unit $\mathbf{V}=(V, \circ, \varepsilon, \oplus, \delta)$ on the set $X\urcorner$. In other words, $V$ supports the free weakly involutive groupoid by defining involution functions that satisfy $(x \circ y)^{\sim}=y^{\sim} \oplus x^{\sim}$. Let $\mathbf{V}_{\circ}$ denote the o-subgroupoid of $\mathbf{V}$ generated by $X\urcorner$ and $\mathbf{V}_{\oplus}$ the $\oplus$-subgroupoid of $\mathbf{V}$ generated by $X\urcorner$. Clearly $\mathbf{V}_{\circ}$ and $\mathbf{V}_{\oplus}$ are isomorphic. Actually, they both support the free involutive groupoid generated by $X$, the first with involutive functions satisfying $(x \circ y)^{\sim}=y^{\sim} \circ x^{\sim}$ and the second with $(x \oplus y)^{\sim}=y^{\sim} \oplus x^{\sim}$. We could have taken sequents as elements of $V_{\circ} \times V_{\oplus}$, but since the two groupoids are isomorphic and support the free involutive groupoid generated by $X$, we chose to identify them.

Lemma 4.8. A classical sequent $x \Rightarrow y$ is valid in $\left(\mathbf{W}_{\mathbf{I n G L}}, \mathbf{F m}\right)$ iff $f^{\circ}(x) \Rightarrow f^{\oplus}(y)$ is provable in $\mathbf{I n G L}$, where $f$ is the identity map.

For an involutive frame $\mathbf{W}$ and $X \subseteq W$, we define $(\sim n) X$ and $X^{\sim n}$ [also $(-n) X$ and $X^{-n}$ ] by $(\sim 0) X=X^{\sim 0}=X,(\sim(n+1)) X=\sim(\sim n) X, X^{\sim(n+1)}=\left(X^{\sim n}\right)^{\sim}$.

Lemma 4.9. Let $\mathbf{W}$ be a residuated frame.

(i) The operation + on $\mathbf{W}^{+}$is order-preserving in both coordinates.

(ii) If $X, Y \in \mathbf{W}^{+}$, then $X^{\triangleleft}+Y^{\triangleleft} \subseteq(X \oplus Y)^{\triangleleft}$.

(iii) If $X \in \mathbf{W}^{+}$, then $(\sim n) X=X^{\sim n}$ and $(-n) X=X^{-n}$, for all even $n \in \mathbb{N}$. 
Proof. (i) For subsets $X_{1}, X_{2}, Y_{1}, Y_{2}$ of $W$ with $X_{1} \subseteq X_{2}$ and $Y_{1} \subseteq Y_{2}$ we have $X_{2}^{\triangleright} \subseteq X_{1}^{\triangleright}$ and $Y_{2}^{\triangleright} \subseteq Y_{1}^{\triangleright}$. Therefore, $X_{2}^{\triangleright} Y_{2}^{\triangleright} \subseteq X_{1}^{\triangleright} Y_{1}^{\triangleright}$ and $\left(X_{1}^{\triangleright} Y_{1}^{\triangleright}\right)^{\triangleleft} \subseteq$ $\left(X_{2}^{\triangleright} Y_{2}^{\triangleright}\right)^{\triangleleft}$, namely $X_{1}+Y_{1} \subseteq X_{2}+Y_{2}$. We used that $X+Y=-[(\sim Y)(\sim X)]=$ $\left(Y^{\triangleright \sim} X^{\triangleright \sim}\right)^{-\triangleleft}=\left(X^{\triangleright \sim-} Y^{\triangleright \sim-}\right)^{\triangleleft}=\left(X^{\triangleright} Y^{\triangleright}\right)^{\triangleleft}$. (ii) Recall that $\triangleleft \triangleright$ is a closure operator, $\triangleleft$ is order-reversing and that $X \circ Y \subseteq X \cdot Y$, for all $X, Y \subseteq W$. Also, we define $X \oplus Y$ element-wise. We have $X^{\triangleleft}+Y^{\triangleleft}=-\left[\left(\sim Y^{\triangleleft}\right) \cdot\left(\sim X^{\triangleleft}\right)\right]=\left(Y^{\triangleleft \triangleright \sim} \cdot X^{\triangleleft \triangleright \sim}\right)^{-\triangleleft} \subseteq$ $\left(Y^{\sim} \cdot X^{\sim}\right)^{-\triangleleft} \subseteq\left(Y^{\sim} \circ X^{\sim}\right)^{-\triangleleft}=(X \oplus Y)^{\triangleleft}$, so $X^{\triangleleft}+Y^{\triangleleft} \subseteq(X \oplus Y)^{\triangleleft}$. (iii) For $w \in W$, we have $w \in \sim X=X^{\triangleright \sim}$ iff $w^{-} \in X^{\triangleright}$ iff $X N w^{-}$. We also have $\sim X=X^{\triangleright \sim} N w$ iff $w^{-} N X^{\triangleright}$ iff $w^{-} \in X^{\triangleright \triangleleft}=X$. Hence $w \in \sim \sim X$ iff $\sim X N w^{-}$iff $w^{--} \in X$ iff $w \in X^{\sim \sim}$. So, $\sim \sim X=X^{\sim \sim}$ and for every even $n,(\sim n) X=X^{\sim n}$.

Theorem 4.10. If $(\mathbf{W}, \mathbf{B})$ is a cut-free involutive Gentzen frame, where $\mathbf{B}$ is a total algebra, then every sequent that is valid in $\mathbf{W}^{+}$is also valid in $(\mathbf{W}, \mathbf{B})$.

Proof. Assume that $s=(x \Rightarrow y)$ is valid in $\mathbf{W}^{+}$and let $f: \mathbf{F m} \rightarrow \mathbf{B}$ be a homomorphism. We will show that $f^{\circ}(x) N f^{\oplus}(y)$. Since $s$ is valid in $\mathbf{W}^{+}$, we have $\bar{f}^{\circ}(x) \subseteq \bar{f}^{\oplus}(y)$, where $\bar{f}: \mathbf{F m} \rightarrow \mathbf{W}^{+}$is the homomorphism that satisfies $\bar{f}(v)=\gamma_{N}(\{f(v)\})$ for each variable $v$.

For brevity we adopt the notation $\neg n=\sim n$ for nonnegative integers and $\neg n=$ $-|n|$ for negative integers. By definition there exist formulas $a_{1}, \ldots, a_{n}, b_{1}, \ldots, b_{m} \in$ $F m$ and groupoid (with unit) terms $t_{x}, t_{y}$ such that $x=t_{x}^{\mathbf{F m}^{i}}\left(a_{1}^{\neg k_{1}}, \ldots, a_{n}^{\neg k_{n}}\right)$ and $y=t_{y}^{\mathbf{F m}^{i}}\left(b_{1}^{\neg l_{1}}, \ldots, b_{m}^{\neg l_{m}}\right)$. Then $\bar{f}^{\circ}(x)=t_{x}^{\left(\mathbf{W}^{+}, \cdot\right)}\left(\left(\neg k_{1}\right) \bar{f}\left(a_{1}\right), \ldots,\left(\neg k_{n}\right) \bar{f}\left(a_{n}\right)\right)$ and $\bar{f}^{\oplus}(y)=t_{y}^{\left(\mathbf{W}^{+},+\right)}\left(\left(\neg l_{1}\right) \bar{f}\left(b_{1}\right), \ldots,\left(\neg l_{n}\right) \bar{f}\left(b_{m}\right)\right)$.

In view of Theorem 4.4. Lemma 3.1 also applies to formulas with negations and it yields $\bar{f}(c) \subseteq\{f(c)\}^{\triangleleft}$ and $f(c) \in \bar{f}(c)$, for all formulas $c \in F m$. From $\bar{f}(c) \subseteq\{f(c)\}^{\triangleleft}$ we also obtain $f(c) \in \bar{f}(c)^{\triangleright}$, so $f(c)^{\sim} \in \bar{f}(c)^{\triangleright \sim}=\sim \bar{f}(c)$ and $f(c)^{-} \in-\bar{f}(c)$. Thus, using Lemma 4.9(iii), we can show that for all $k$ we have

$$
f(c)^{\neg k} \in(\neg k) \bar{f}(c) .
$$

So,

$$
\begin{aligned}
f^{\circ}(x) & =f^{\circ}\left(t_{x}^{\mathbf{F m}^{i}}\left(a_{1}^{\neg k_{1}}, \ldots, a_{n}^{\neg k_{n}}\right)\right) & & \\
& =t_{x}^{(W, \circ)}\left(f\left(a_{1}\right)^{\neg k_{1}}, \ldots, f\left(a_{n}\right) \neg k_{n}\right) & & \left(f^{\circ} \text { extends } f\right) \\
& \in t_{x}^{(\mathcal{P}(W) \circ \circ)}\left(\left(\neg k_{1}\right) \bar{f}\left(a_{1}\right), \ldots,\left(\neg k_{n}\right) \bar{f}\left(a_{n}\right)\right) & & (*),(\circ \text { in } \mathcal{P}(W) \text { is element-wise }) \\
& \subseteq t_{x}^{\left(\mathbf{W}^{+}, .\right)}\left(\left(\neg k_{1}\right) \bar{f}\left(a_{1}\right), \ldots,\left(\neg k_{n}\right) \bar{f}\left(a_{n}\right)\right) & & \left(\gamma_{N} \text { is a closure operator }\right) \\
& =\bar{f}^{\circ}(x) . & &
\end{aligned}
$$

From $f(c) \in \bar{f}(c)$ we have $\{f(c)\}\urcorner \subseteq \bar{f}(c)\urcorner$; hence $\bar{f}(c)\urcorner \triangleleft \subseteq\{f(c)\}\urcorner \triangleleft$, namely $\neg \bar{f}(c) N\{f(c)\}\urcorner$. By the negation rules for $N$, we have $(\neg \bar{f}(c))^{\neg k} N\{f(c)\} \neg(\neg k)$ for every even integer $k$, so $(\neg \bar{f}(c))^{k} \subseteq\left\{f(c)^{\neg(k+1)}\right\}^{\triangleleft}$. Using Lemma 4.9(iii), we have $(\neg(k+1)) \bar{f}(c) \subseteq\left\{f(c)^{\neg(k+1)}\right\} \triangleleft$, for every even integer $k$.

On the other hand, from $\bar{f}(c) \subseteq\{f(c)\}^{\triangleleft}$ we have $\bar{f}(c) N f(c)$. By the negation rules for $N$, we have $\bar{f}(c)^{\neg k} N f(c)^{\neg k}$, for every even integer $k$, so $\bar{f}(c)^{\neg k} \subseteq$ $\left\{f(c)^{(\neg k)}\right\}^{\triangleleft}$. In view of Lemma 4.9 (iii), we obtain $(\neg k) \bar{f}(c) \subseteq\left\{f(c)^{(\neg k)}\right\}^{\triangleleft}$, for every even integer $k$. Consequently, we have

$$
(\neg k) \bar{f}(c) \subseteq\left\{f(c)^{(\neg k)}\right\}^{\triangleleft}, \text { for every integer } k .
$$




$$
\begin{array}{rlrl}
\bar{f}^{\oplus}(y) & =t_{y}^{\left(\mathbf{W}^{+},+\right)}\left(\left(\neg l_{1}\right) \bar{f}\left(b_{1}\right), \ldots,\left(\neg l_{n}\right) \bar{f}\left(b_{m}\right)\right) & \\
& \subseteq t_{y}^{\left(\mathbf{W}^{+},+\right)}\left(f\left(b_{1}\right)^{\left(\neg l_{1}\right) \triangleleft}, \ldots, f\left(b_{m}\right)^{\left(\neg l_{m}\right) \triangleleft}\right) & & (* *),(\text { Lem. 4.9.9(i)) } \\
& =\left[t_{y}^{(W, \oplus)}\left(f\left(b_{1}\right)^{\left(\neg l_{1}\right)}, \ldots, f\left(b_{m}\right)^{\left(\neg l_{m}\right)}\right)\right]^{\triangleleft}=\left\{f^{\oplus}(y)\right\}^{\triangleleft} & & (\text { Lemma 4.9)(ii)). }
\end{array}
$$

So $f^{\circ}(x) \in \bar{f}^{\circ}(x) \subseteq \bar{f}^{\oplus}(y) \subseteq\left\{f^{\oplus}(y)\right\}^{\triangleleft}$; thus $f^{\circ}(x) \in\left\{f^{\oplus}(y)\right\}^{\triangleleft}$, i.e., $f^{\circ}(x) N f^{\oplus}(y)$.

Corollary 4.11 (Adequacy). If a sequent is valid in $\mathrm{InGL}$, then it is valid in all cut-free involutive Gentzen frames.

Combining the soundness of InGL (InFL) given by Lemma 4.1, and their adequacy given as part of Corollary 4.11, we have the completeness of these systems.

Corollary 4.12 (Completeness). A sequent is provable in InGL iff it is valid in RLUG. The same holds for InFL and InFL.

A cut-free system for InFL-algebras is presented in [1. A lengthy syntactic argument is given to establish cut elimination for this system, and decidability follows readily. We give a brief argument below and subsequently prove the stronger FMP. For cyclic InFL-algebras, a cut-free system and a decision procedure are given in $[26$.

Corollary 4.13. The systems InGL and InFL enjoy the cut-elimination property.

Proof. Every sequent provable in InGL, is valid in $\operatorname{lnGL}$; hence it is also valid in the frame associated with cut-free InGL, by Corollary 4.11] Thus it is provable in cut-free InGL. Conversely, every sequent provable in cut-free InGL is obviously provable in InGL.

Decidability. Even though an exhaustive proof search for a given sequent in InGL or InFL is never finite, we can still restrict our attention to a finite part.

Theorem 4.14. The equational theories of InFL and InGL-algebras are decidable.

Proof. At every step of a proof search there are only finitely many sequents, obtained by applying $(\sim)$ and $\left(^{-}\right)$, such that not all formulas have external negations on them, and these sequents are easily identified; for a description of how to obtain all such sequents, see the figure below. If all formulas have external negations, then no logical rule can be applied (upwards). Therefore we need to explore, by using $(\sim)$ and $\left(^{-}\right)$, only finitely many sequents between applications of logical rules, namely among the sequents obtained by applying $\left({ }^{\sim}\right)$ and $\left(^{-}\right)$to our current sequent only those where not all formulas have external negations plus the ones obtained in the process of getting to such sequents (via the figure below). Note that upward applications of logical rules decrease the number of connectives in the involved sequents.

Indeed, for the associative case, if $a_{1} \circ a_{2} \circ \cdots \circ a_{m} \Rightarrow b_{1} \circ b_{2} \circ \cdots \circ b_{n}$ is a sequent, then the possible sequents that can be obtained using the rules for external negations are given by moving the formulas according to the following diagram:

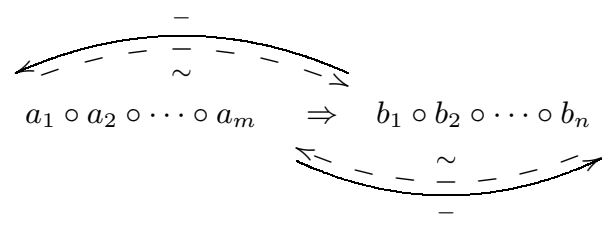

In the nonassociative case the possible moves are even more restricted; therefore we obtain a finite set of possible sequents with at least one nonnegated formula. 
The (unital) involutive frames we have defined satisfy the property: $x N y^{-}$iff y $N x^{\sim}$ (Lemma 4.2(i)). Also the following rule (followed by its derivation) holds in InGL.

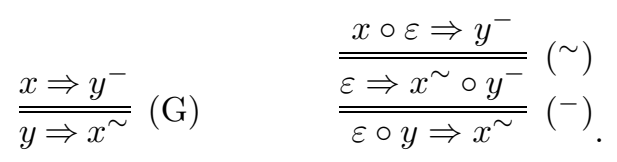

Cut elimination with simple structural rules. We will extend simple structural rules to the involutive case. Let $t_{0}, t_{1}, \ldots, t_{n}$ be elements of the free groupoid with unit (using the signature of $\{0, \varepsilon\}$ ) over a countable set of possibly negated variables, i.e., formal expressions of the form $p^{\urcorner n}$, for even integers $n$, with the usual conventions for $\neg$ adopted in the proof of Theorem 4.10. The construction is similar to $\mathrm{Fm}^{i}$. We also assume that $t_{0}$ is linear. A simple rule allowing negations is of the form

$$
\frac{t_{1} N q \quad \cdots \quad t_{n} N q}{t_{0} N q}(\mathrm{r})
$$

where $q$ is a variable not occurring in $t_{0}, t_{1}, \ldots, t_{n}$; a simple rule is one where $n=0$ in all $p^{\neg n}$ above. The definition of a simple rule in InGL, satisfaction of a rule in a frame and the correspondence between simple rules (allowing negations) and equations are as before. So, a simple equation allowing negations over $\{\vee, \cdot, 1, \sim,-\}$ is of the form $t_{0}^{\mathbf{F m}} \leq t_{1}^{\mathbf{F m}} \vee \cdots \vee t_{n}^{\mathbf{F m}}$, where $t_{0}$ is linear and negations are applied directly an even number of times to the variables.

Theorem 4.15. Let $(\mathbf{W}, \mathbf{B})$ be a cut-free involutive Gentzen frame and let e be a simple equation allowing negations. Then $(\mathbf{W}, \mathbf{B})$ satisfies $\mathrm{R}(e)$ iff $\mathbf{W}^{+}$satisfies e.

Proof. Let $e$ be of the form $t_{0}^{\mathbf{F m}} \leq t_{1}^{\mathbf{F m}} \vee \cdots \vee t_{n}^{\mathbf{F m}}$, where $t_{0}$ is linear. As in Theorem 4.10, $e^{\mathbf{W}^{+}}(\bar{X})$ holds iff $t_{0}^{\mathbf{W}^{+}}(\bar{X}) \subseteq t_{1}^{\mathbf{W}^{+}}(\bar{X}) \vee \cdots \vee t_{n}^{\mathbf{W}^{+}}(\bar{X})$, for all sequences $\bar{X}=\left(X_{j}\right)_{j \in J}$ of elements in $\mathbf{W}^{+}$. By Lemma 1.2(iv), this is equivalent to the stipulation that for all $z \in W^{\prime}, t_{1}^{\mathbf{W}^{+}}(\bar{X}) \vee \cdots \vee t_{n}^{\mathbf{W}^{+}}(\bar{X}) \subseteq\{z\}^{\triangleleft}$ implies $t_{0}^{\mathbf{W}^{+}}(\bar{X}) \subseteq$ $\{z\}^{\triangleleft}$. As $t_{1}^{\mathbf{W}^{+}}(\bar{X}) \vee \cdots \vee t_{n}^{\mathbf{W}^{+}}(\bar{X})=\gamma_{N}\left(t_{1}^{\mathbf{W}^{+}}(\bar{X}) \cup \cdots \cup t_{n}^{\mathbf{W}^{+}}(\bar{X})\right), t_{i}^{\mathbf{W}^{+}}(\bar{X})=$ $\gamma_{N}\left(t_{i}^{\mathcal{P}(\mathbf{W})}(\bar{X})\right)$ and in view of Lemma $4.9($ iii), this is equivalent to asking that $(*)$

$$
\forall z \in W^{\prime} \text {, if } t_{i}^{\mathcal{P}(\mathbf{W})}(\bar{X}) \subseteq\{z\}^{\triangleleft} \text { for every } i \in\{1, \ldots, n\}, \text { then } t_{0}^{\mathcal{P}(\mathbf{W})}(\bar{X}) \subseteq\{z\}^{\triangleleft} .
$$

Assume first that $(\mathbf{W}, \mathbf{B})$ satisfies $\mathrm{R}(e)$ and let $x_{j} \in X_{j}$, for all $j \in J$ (we write $\bar{x} \in \bar{X}$ and $\left.\bar{x}=\left(x_{j}\right)_{j \in J}\right)$. Note that $t_{i}^{\mathbf{W}}(\bar{x})=t_{i}^{\mathbf{W}}\left(\left(x_{j}\right)_{j \in J}\right) \in t_{i}^{\mathcal{P}(\mathbf{W})}\left(\left(\left\{x_{j}\right\}\right)_{j \in J}\right) \subseteq$ $t_{i}^{\mathcal{P}(\mathbf{W})}(\bar{X})$, by the definition of the operations in $\mathcal{P}(\mathbf{W})$. Note further that for all $z \in W^{\prime}$, if $t_{i}^{\mathcal{P}(\mathbf{W})}(\bar{X}) \subseteq\{z\}^{\triangleleft}$ for every $i \in\{1, \ldots, n\}$, then $t_{i}^{\mathbf{W}}(\bar{x}) N z$, for all $i \in\{1, \ldots, n\}, t_{0}^{\mathbf{W}}(\bar{x}) N z$, by $\mathrm{R}(e)$, and $t_{0}^{\mathbf{W}}(\bar{x}) \in\{z\}^{\triangleleft}$, for all $\bar{x} \in \bar{X}$. Since $t_{0}$ is a linear term, we obtain $t_{0}^{\mathcal{P}(\mathbf{W})}(\bar{X}) \subseteq\{z\}^{\triangleleft}$, namely $(*)$ holds and $\mathbf{W}^{+}$satisfies $e$.

Conversely, assume that $(*)$ holds for $X_{j}=\gamma_{N}\left(\left\{x_{j}\right\}\right)$, where $x_{j} \in W$; namely for all $z \in W^{\prime},\left\{t_{1}^{\mathbf{W}}(\bar{x}), \ldots, t_{n}^{\mathbf{W}}(\bar{x})\right\} \subseteq\{z\}^{\triangleleft}$ implies $t_{0}^{\mathbf{W}}(\bar{x}) \in\{z\}^{\triangleleft}$. Consequently, $\left(t_{1}^{\mathbf{W}}(\bar{x}) N z\right.$ and $\left.\ldots t_{n}^{\mathbf{W}}(\bar{x}) N z\right)$ implies $t_{0}^{\mathbf{W}}(\bar{x}) N z$, and $\mathrm{R}(e)$ holds in $(\mathbf{W}, \mathbf{B})$.

Corollary 4.16. All simple rules allowing negations are preserved by $\left({ }_{-}\right)^{+}$. Consequently, all extensions of InGL by such rules enjoy the cut-elimination property. 


\section{FinITE MODEL PROPERTY FOR InGL AND EXTENSIONS}

Since $\sim$ is a bijection, every finite involutive $r \ell u$-groupoid satisfies the equation $(\sim n) x=x$ for some natural number $n$, which is equivalent to the equation $x=$ $(-n) x$. If $n$ is an odd number, then $f(x)=(\sim n) x$ is an order antimorphism, so the involutive $r \ell u$-groupoid is trivial. Consequently, every finite nontrivial involutive $r \ell u$-groupoid satisfies an equation of the form $(\sim n) x=x$, and $n$ can be taken to be both even and minimal nonzero. Clearly $(\sim 2) x=x$ is equivalent to cyclicity $\sim x=-x$. Consider the following bi-directional rule:

$$
\frac{x^{\sim n} \Rightarrow z}{x \Rightarrow z}(\sim n)
$$

We will call two sequences of formulas $n$-equivalent if one is obtained from the other by adding or removing exactly $n$-many negations (of the same kind) to/from some of the formulas in the sequence. Clearly this equivalence (in the presence of the external negations rules) simulates the effect of the rule $(\sim n)$ to the one side of the sequents involved. We extend the equivalence also to sequents in the obvious way. For every equivalence class there are representatives such that all formulas in them contain no more than $n$ negations. We will call them minimal representatives. The next result follows from Theorem 4.15.

Corollary 5.1. Let $\mathbf{W}$ be an involutive frame for InGL and $n$ an even natural number. The frame $\mathbf{W}$ satisfies the rule $(\sim n)$ iff $\mathbf{W}^{+}$satisfies $(\sim n) x=x$.

Corollary 5.2. The system InGL $+(\sim n)$ has the cut-elimination property, for every even natural number $n$. Furthermore, it is decidable.

Proof. Soundness of the calculus is routine. The proof proceeds as for the cut elimination of InGL, by using Corollary 5.1. Decidability follows from the observation that, due to the rule $(\sim n)$, there are finitely many sequents with up to $n$ negations that need to be considered at each step of the proof search.

Corollary 5.3. InGL $+(\sim n)$ has the FMP, for every even natural number $n$.

Proof. We consider the involutive frame $\mathbf{W}$, where $W$ is the free groupoid over $F m$ and $x N z$ iff $\vdash_{\text {InGL }+(\sim n)} x \Rightarrow z$. Consider a sequent $s$ that is not provable and let $s^{\leftarrow}$ be the set of all the sequents involved in a complete proof search for $s$. Note that $s^{\leftarrow}$ is infinite, since the $(\sim)$ and $\left(^{-}\right)$rules can be applied an arbitrary number of times. As in Theorem 3.15, we can show that $N^{\prime}=N \cup\left(s^{\leftarrow}\right)^{c}$ is a nuclear relation and $\mathbf{W}^{\prime}=\left(W, N^{\prime}, \circ,\{\varepsilon\}, \sim,{ }^{-}\right)$is an involutive frame for $\mathbf{I n G L}+(\sim n)$. Clearly $s^{\leftarrow}$ is a union of $n$-equivalence classes and there are only finitely many such classes. If $y, z \in W$ are $n$-equivalent, then $\{y\}^{\triangleleft}=\{z\}^{\triangleleft}$ because of the rule $(\sim n)$. So, the basic closed sets are finitely many and $\mathbf{W}^{\prime+}$ is finite. Moreover, $s$ fails in $\mathbf{W}^{\prime+}$.

For $x \in W$ and $m \in \mathbb{Z}^{+}, x^{\neg m}$ is $x^{\sim m}$ if $m>0, x^{-m}$ if $m<0$, and $x$ if $m=0$.

Theorem 5.4. Every sequent provable in InGL $+(\sim n)$ has a proof in InGL augmented by initial sequents of the form $a \Rightarrow a^{\neg n}$, where $k \in \mathbb{Z}$.

Proof. If a sequent $s$ is provable in $\mathbf{I n G L}+(\sim n)$, it is provable in the cut-free system, by Corollary 5.2. All of the systems we will mention in this proof will be 
considered in their cut-free versions. In the presence of $(\sim)$ and $\left(^{-}\right)$, the rules

$$
\frac{u(x) \Rightarrow z}{\overline{u\left(x^{\sim n}\right) \Rightarrow z}}(\sim n \mathrm{~L}) \quad \frac{x \Rightarrow u(z)}{\overline{x \Rightarrow u\left(z^{\sim n}\right)}}(\sim n \mathrm{R})
$$

are derivable from $(\sim n)$, and vice versa. Here $u$ is such that $u(x)$ is an element of $W$ in which no negations are applied to $x$. Note that these rules could have been called $(-n \mathrm{~L})$ and $(-n \mathrm{R})$, as the versions with - instead of $\sim$ are simply the upward direction of the rule, since $x^{-\sim}=x$. So, $s$ is provable in $\mathbf{I n G L}+(\sim n \mathrm{~L})+(\sim n \mathrm{R})$.

We will first prove, inductively, that the rules $(\sim n \mathrm{~L})$ and $(\sim n \mathrm{R})$ can be moved to the top of the proof in $\mathbf{I n G L}+(\sim n \mathrm{~L})+(\sim n \mathrm{R})$, namely that there is a proof of $s$ in which all applications of the rules $(\sim n \mathrm{~L})$ and $(\sim n \mathrm{R})$ precede all applications of rules in InGL. We proceed by focusing on the rule applied immediately before $(\sim n \mathrm{~L})$ or $(\sim n \mathrm{R})$. Below, we give a proof and its rewritten version. We will often be using the following instances of $\left({ }^{\sim}\right)$ and $\left(^{-}\right)$, which hold for all even $n$ :

$$
\frac{x \Rightarrow z^{-n}}{\overline{x^{\sim n} \Rightarrow z}}\left({ }_{n}\right) \quad \frac{x^{-n} \Rightarrow z}{\overline{x \Rightarrow z^{\sim n}}}\left({ }^{-} n\right) .
$$

We first deal with the case where $(\sim n \mathrm{~L})$, applied upward, is preceded by a left rule. For $(\sim \mathrm{L})$, we have

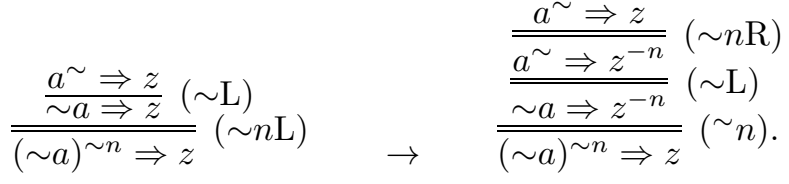

Note that this case illustrates the necessity of replacing $(\sim n)$ by $(\sim n \mathrm{~L})$, and $(\sim n \mathrm{R})$, in our arguments. If $\sim n$ is applied to $x$, the situation is even simpler. The rewriting for $(-\mathrm{L})$ and the other left rules is similar. For example, for $(\mathrm{VL})$, we have

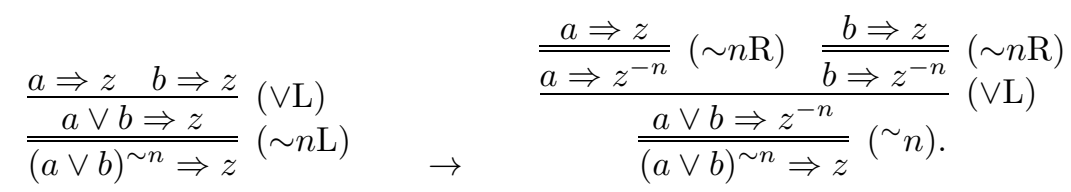

For the case where $(\sim n \mathrm{R})$ is preceded by a right rule, of interest are $(\sim \mathrm{R})$ and $(\cdot \mathrm{R})$ :

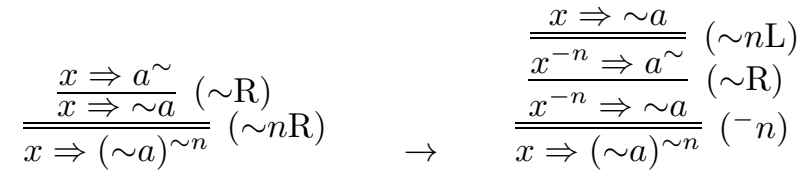

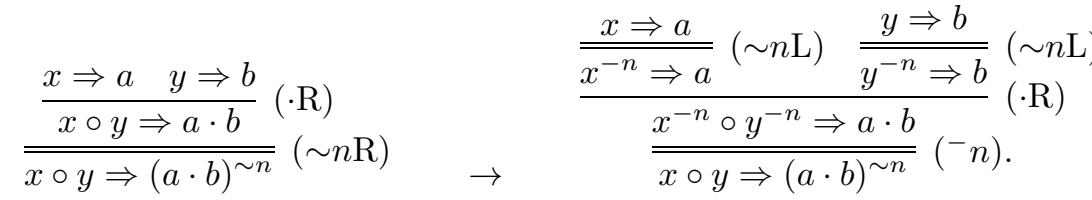

We used $(x \circ y)^{-n}=x^{-n} \circ y^{-n}$, which is true for even $n$. The remaining right rules are handled in the same way. The cases where $(\sim n \mathrm{R})$ is preceded by a left rule and $(\sim n \mathrm{~L})$ is preceded by a right rule are much simpler. We show only one example:

$$
\begin{array}{ll}
\frac{x \Rightarrow a}{x \Rightarrow a \vee b}(\vee \mathrm{L}) & \frac{x \Rightarrow a}{\overline{x^{\sim n} \Rightarrow a}}(\sim n \mathrm{~L}) \\
\overline{x^{\sim n} \Rightarrow a \vee b} & (\sim n \mathrm{R}) \quad \rightarrow \quad \frac{}{x^{\sim n} \Rightarrow a \vee b}(\vee \mathrm{R}) .
\end{array}
$$


So far we have considered the bidirectional rules $(\sim n \mathrm{~L})$ and $(\sim n \mathrm{R})$ in the downward direction. In the inverse direction they take the same form, but with $\sim n$ replaced by ${ }^{-n}$; this shows that the rules could have been called $(-n \mathrm{~L})$ and $(-n \mathrm{R})$. The proof rewriting for these cases is completely analogous to the cases handled above.

Finally, we show that $(\sim n \mathrm{~L})$ and $(\sim n \mathrm{R})$ commute with the rules $(\sim)$ and $\left(^{-}\right)$. We show two illustrative cases. We use $\left(y^{\sim n}\right)^{-}=\left(y^{-}\right)^{\sim n}$, and $u(y)^{-}=u^{-}\left(y^{-}\right)$, where $u^{-}$is obtained from $u$ by reversing the order and applying - to every factor:

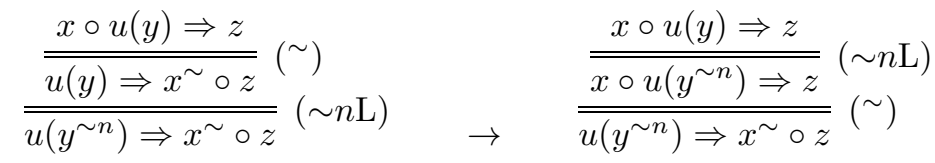

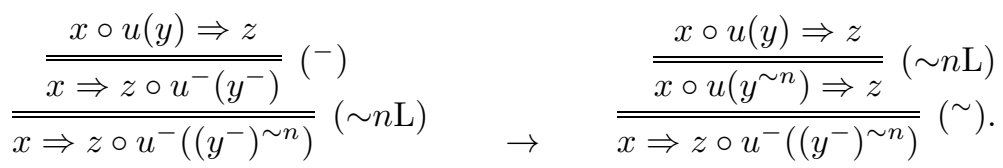

We have shown that there is a proof of the sequent $s$ in which $(\sim n \mathrm{~L})$ and $(\sim n \mathrm{R})$ are applied before rules of InGL. As the only initial sequents are of the form $a \Rightarrow a$, $\varepsilon \Rightarrow 1$ and $0 \Rightarrow \varepsilon$, the sequents obtained by applications of only $(\sim n \mathrm{~L})$ and $(\sim n \mathrm{R})$ are of the form $a^{\neg m n} \Rightarrow a^{\neg k n}, \varepsilon \Rightarrow 1^{\neg m n}$ and $0^{\neg k n} \Rightarrow \varepsilon$, where $k, m \in \mathbb{Z}$. The first type of sequents are equivalent over InGL to sequents of the form $a \Rightarrow a^{\neg k n}$, for $k \in \mathbb{Z}$; Since $\varepsilon^{\sim}=\varepsilon^{-}=\varepsilon$ the last two sequents are already derivable in InGL. Consequently, we have obtained a proof of $s$ in InGL from these initial sequents.

Corollary 5.5. Given a sequent that is not provable in InGL, there is an upper bound on the number $n$ such that the sequent is provable in $\mathbf{I n G L}+(\sim n)$.

Proof. We define the negation depth $n_{a}(x)$ of an occurrence of a formula $a$ in $x$ inductively, by $n_{a}(a)=0$, and if the formula $b$ contains that occurrence of $a$, then $n_{a}(\sim b)=n_{a}(b)+1, n_{a}(-b)=n_{a}(b)-1, n_{a}(b \bullet c)=n_{a}(b), n_{a}(c \bullet b)=n_{a}(b)$, for - $\in\{\wedge, \vee, \cdot\}$. If $x$ contains that occurrence of $a$, then $n_{a}\left(x^{\sim}\right)=n_{a}(x)+1, n_{a}\left(x^{-}\right)=$ $n_{a}(x)-1, n_{a}(x \circ y)=n_{a}(x), n_{a}(y \circ x)=n_{a}(x)$. For example $n_{a}\left(a^{\urcorner n}\right)=k n$. Note that the subscript $a$ denotes a specific occurrence of the formula $a$, but for the sake of notational simplicity the position of the formula is not made explicit. Given two disjoint occurrences of formulas $a$ and $b$ in a sequent $s$, we define the negation difference $d_{a, b}(s)$ from $b$ to $a$ in $s$ as follows. If $a$ appears in $x$ and $b$ appears in $y$, we define $d_{a, b}(x \Rightarrow y)=d_{a, b}(y \Rightarrow x)=n_{b}(y)-n_{a}(x), d_{a, b}(x \circ y \Rightarrow z)=d_{a, b}(z \Rightarrow$ $y \circ x)=n_{b}(y)-n_{a}(x)-1, d_{a, b}(y \circ x \Rightarrow z)=d_{a, b}(z \Rightarrow x \circ y)=n_{b}(y)-n_{a}(x)+1$. For the situations where $a$ and $b$ appear in the same formula in $s$ with $a$ appearing in $c$ and $b$ appearing in $d$ (we consider fixed occurrences of $c, d$ ): in case the product $c \cdot d$ appears in $s$, we define $d_{a, b}(s)=n_{b}(d)-n_{a}(c)-1$ and in case the product $d \cdot c$ appears in $s$, we define $d_{a, b}(s)=n_{b}(d)-n_{a}(c)+1$. In all other cases, $d_{a, b}(s)$ is undefined (or we can define it to be equal to 0 , as it makes no difference). For example, $\left.d_{a, b}(a \Rightarrow b\urcorner^{k n}\right)=k n$; also the negation difference from the second to the first occurrence of $a$ in $a \Rightarrow a^{\urcorner k n}$ is $k n$. As $d_{a, b}(s)=-d_{b, a}(s)$, it is the absolute values of negation differences that are of interest.

It is straightforward that for each rule of cut-free InGL and for each formula that appears in the numerator of a rule, the maximum negation difference between occurrences of this formula in the sequents in the numerator is less than or equal to 
the maximum negation difference between occurrences of this formula in the denominator of the rule. (Actually, in view of the strong form of the subformula property in cut-free InGL, every occurrence of a formula in a sequent in the numerator of a rule has a corresponding occurrence in the denominator of the rule. The negation difference between two occurrences of a formula in any sequent of the numerator is equal to the negation difference between the two corresponding occurrences of the formula in the denominator.) In short, the rules of cut-free InGL do not decrease negation differences between occurrences of a formula.

Now let $s$ be a sequent, not provable in InGL but provable in InGL $+(\sim n)$. By Theorem 5.4 an axiom of the form $a \Rightarrow a^{\neg k n}$ is used in its proof in the augmented InGL. Let $d$ be the maximum negation difference between any two occurrences of any formula in $s$. The absolute value of the negation difference between the occurrences of $a$ in $a \Rightarrow a^{\neg k n}$ is $|k| n$ for some $k \in \mathbb{Z}$, so $|k| n \leq d$ and $n \leq d /|k|$.

Corollary 5.6. For every sequent $s$ there exists an even natural number $n_{s}$ such that $s$ is provable in $\mathbf{I n G L}+\left(\sim n_{s}\right)$ iff $s$ is provable in $\mathbf{I n G L}$.

Corollary 5.7. The system InGL has the FMP.

Proof. If $s$ is not provable in InGL, it is not provable in InGL $+\left(\sim n_{s}\right)$. The latter has FMP, so $s$ fails in a finite involutive $r \ell u$-groupoid (satisfying $x^{\sim n_{s}}=x$ ).

Lemma 5.8. Rules $(\sim n \mathrm{~L})$ and $(\sim n \mathrm{R})$ commute with all simple structural rules.

Proof. Let (r) be a (multiple conclusion) simple structural rule and assume that $x$ appears in $t_{0}$. We will write $t_{0}(x)$ for $t_{0}$, treating $t_{0}$ as a unary linear polynomial.

$$
\begin{aligned}
& \frac{t_{1} \Rightarrow z \quad \cdots \quad t_{k} \Rightarrow z}{t_{0} \Rightarrow z}(r)
\end{aligned}
$$

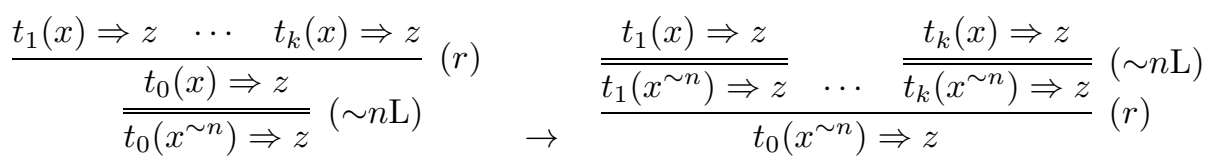

where the uppermost steps in the second proof indicate repeated applications of $(\sim n \mathrm{~L})$ to each occurrence of $x$ in $t_{i}(x)$.

Corollary 5.9. The systems $\mathbf{I n G L}_{\mathrm{R}}$ have the FMP, for every set $\mathrm{R}$ of simple rules (without negations) for which the complexity does not increase. In particular, $\mathbf{I n G L} \mathbf{L}_{a}$ and $\mathbf{I n G \mathbf { L } _ { a e } \text { (or equivalently } \mathbf { I n F L } \text { and } \mathbf { I n F L }} \mathbf{L}_{e}$, resp.) have the FMP.

Proof. First we note that we can only focus on simple rules for which $t_{0}$ contains all of the variables of the rule. If this fails and in the rule $(r) t_{i}$ contains a variable $x$ not in $t_{0}$, then the rule $\left(r^{\prime}\right)$ obtained from $(r)$ by deleting the sequent containing $t_{i}$ is equivalent to $(r)$, as semantically we can make the value of $x$, and therefore also of $t_{i}$, arbitrarily small, which means that $t_{i}$ is redundant in the corresponding equation $t_{0}^{\mathbf{F m}} \leq t_{1}^{\mathbf{F m}} \vee \cdots \vee t_{n}^{\mathbf{F m}}$. Consider thus an instance of a rule where every variable $x$ of the numerator also appears in the denominator. For every formula in any sequent in the numerator, the maximum negation difference between occurrences of the formula in this sequent in the numerator is less than or equal to the maximum negation difference between occurrences of the formula in the denominator. This follows from the observation that if the two occurrences of the formula in the sequent in the numerator appear in instances of the (possibly identical) variables $x$ and $y$, and since $x$ and $y$ will also appear in the denominator, then the absolute value of 
the negation difference will also be witnessed in the denominator. Consequently, the maximum of the absolute values of negation differences (of two occurrences of a formula) does not decrease downward in an application of a simple rule. Hence the reasoning in the proof of Corollary 5.5 applies in view of Lemma 5.8 ,

We mention that the preceding results can also be reformulated with an equivalent one-sided sequent calculus for InFL. Finally, we would like to thank the anonymous referee for many valuable comments and corrections.

\section{REFERENCES}

[1] V. M. Abrusci, Phase semantics and sequent calculus for pure noncommutative classical linear propositional logic, J. Symbolic Logic 56 (1991), no. 4, 1403-1451. MR1136467 (93a:03060)

[2] F. Belardinelli, P. Jipsen, and H. Ono, Algebraic aspects of cut elimination, Studia Logica 77 (2004), 209-240. MR2080239 (2005d:03039)

[3] W. J. Blok and C. J. van Alten, The finite embeddability property for residuated lattices, pocrims and BCK-algebras, Algebra Universalis 48 (2002), 253-271. MR 1954775 (2003j:06017)

[4] W. J. Blok and C. J. van Alten, On the finite embeddability property for residuated ordered groupoids, Trans. Amer. Math. Soc. 357 (2005), no. 10, 4141-4157. MR2159703 (2007b:06019)

[5] K. Blount, On the structure of residuated lattices, Ph.D. thesis, Vanderbilt University, Nashville, Tennessee, 1999. MR2700270

[6] K. Blount and C. Tsinakis, The structure of residuated lattices, International Journal of Algebra and Computation 13 (2003), no. 4, 437-461. MR2022118(2005b:06011)

[7] W. Buszkowski, Interpolation and FEP for logics of residuated algebras, Log. J. of IGPL. doi:10.1093/jigpal/jzp094.

[8] A. Ciabattoni, N. Galatos, and K. Terui, From axioms to analytic rules in nonclassical logics, Proceedings of LICS'08 (2008), 229-240.

[9] A. Ciabattoni, N. Galatos, and K. Terui, The expressive power of structural rules for FL. submitted.

[10] J. M. Dunn, M. Gehrke, and A. Palmigiano, Canonical extensions and relational completeness of some substructural logics, J. Symbolic Logic 70 (2005), no. 3, 713-740. MR 2155263 (2006f:06012)

[11] M. Farulewski, Finite embeddability property for residuated groupoids, Rep. Math. Logic 43 (2008), 25-42. MR.2417720 (2009e:03066)

[12] N. Galatos, P. Jipsen, T. Kowalski, and H. Ono, Residuated lattices: an algebraic glimpse at substructural logics, Studies in Logic and the Foundations of Mathematics, vol. 151, Elsevier, 2007. MR2531579 (2011d:03033)

[13] N. Galatos and H. Ono, Algebraization, parametrized local deduction theorem and interpolation for substructural logics over FL, Studia Logica 83 (2006), 279-308. MR 2250112 (2007d:03035)

[14] N. Galatos and H. Ono, Cut elimination and strong separation for substructural logics: an algebraic approach, Annals of Pure and Applied Logic 161 (2010), 1097-1133. MR2646809

[15] N. Galatos and C. Tsinakis, Generalized MV-algebras, J. of Algebra 283 (2005), 254-291. MR2102083 (2005h:06012)

[16] B. Ganter and R. Wille, Formal Concept Analysis: Mathematical Foundations, SpringerVerlag, Berlin, 1998. MR.1707295 (2000i:06002b)

[17] M. Gehrke, Generalized Kripke frames, Studia Logica 84 (2006), 241-275. MR 2284541 (2007j:03031)

[18] P. Jipsen and C. Tsinakis, A survey of residuated lattices, Ordered Algebraic Structures (J. Martinez, ed.), Kluwer, Dordrecht, 2002, pp. 19-56. MR2083033(2005e:06024)

[19] M. Okada and K. Terui, The finite model property for various fragments of intuitionistic linear logic, Journal of Symbolic Logic 64 (1999), 790-802. MR.1777787 (2002g:03043)

[20] H. Ono and Y. Komori, Logics without the contraction rule, Journal of Symbolic Logic 50 (1985), 169-201. MR780534 (87a:03053) 
[21] K. I. Rosenthal, Quantales and Their Applications, Pitman Research Notes in Mathematics, vol. 234, Longman, 1990. MR1088258 (92e:06028)

[22] J. Schmidt and C. Tsinakis, Relative pseudo-complements, join-extensions and meetretractions, Mathematische Zeitschrift 157 (1977), 271-284. MR0472618 (57:12314)

[23] K. Terui, Which Structural Rules Admit Cut Elimination? An Algebraic Criterion, Journal of Symbolic Logic 72 (2007), no. 3, 738-754. MR2354898 (2008h:03063)

[24] C. J. van Alten, The finite model property for knotted extensions of propositional linear logic, Journal of Symbolic Logic 70 (2005), no. 1, 84-98. MR.2119124 (2005m:03127)

[25] C. J. van Alten and J. G. Raftery, Rule Separation and Embedding Theorems for Logics Without Weakening, Studia Logica 76 (2004), 241-274. MR2072985(2005e:03132)

[26] A. M. Wille, A Gentzen system for involutive residuated lattices, Algebra Universalis 54 (2005), no. 4, 449-463. MR2218856 (2007j:03092)

[27] D. N. Yetter, Quantales and (noncommutative) linear logic, J. Symbolic Logic 55 (1990), no. 1, 41-64. MR.1043543 (91f:03052)

Department of Mathematics, University of Denver, 2360 S. Gaylord Street, Denver, COLORADO 80208

E-mail address: ngalatos@du.edu

Mathematics and CS, Faculty of Mathematics, School of Computer Science, Chapman

University, One University Drive, Orange, California 92866

E-mail address: jipsen@chapman.edu 